\title{
Radius Margin Bounds for Support Vector Machines with the RBF Kernel
}

\author{
Kai-Min Chung \\ b88061@csie.ntu.edu.tw \\ Wei-Chun Kao \\ b89106@csie.ntu.edu.tw \\ Chia-Liang Sun \\ b88047@csie.ntu.edu.tw \\ Li-Lun Wang \\ b7506054@csie.ntu.edu.tw \\ Chih-Jen Lin \\ lincj@ntu.edu.tw \\ Department of Computer Science and Information Engineering, \\ National Taiwan University, Taipei 106, Taiwan
}

An important approach for efficient support vector machine (SVM) model selection is to use differentiable bounds of the leave-one-out (loo) error. Past efforts focused on finding tight bounds of loo (e.g., radius margin bounds, span bounds). However, their practical viability is still not very satisfactory. Duan, Keerthi, and Poo (2003) showed that radius margin bound gives good prediction for L2-SVM, one of the cases we look at. In this letter, through analyses about why this bound performs well for L2SVM, we show that finding a bound whose minima are in a region with small loo values may be more important than its tightness. Based on this principle, we propose modified radius margin bounds for L1-SVM (the other case) where the original bound is applicable only to the hard-margin case. Our modification for L1-SVM achieves comparable performance to L2-SVM. To study whether L1- or L2-SVM should be used, we analyze other properties, such as their differentiability, number of support vectors, and number of free support vectors. In this aspect, L1-SVM possesses the advantage of having fewer support vectors. Their implementations are also different, so we discuss related issues in detail.

1 Introduction

Recently, support vector machines (SVM) (Vapnik, 1998) have been a promising tool for data classification. Their success depends on the tuning of several parameters that affect the generalization error.

The generalization error can be estimated by, for example, testing some data that are not used for training; cross validation and leave-one-out (loo) 
estimate the error in this way. Loo is particularly of theoretical interest, because it makes use of the greatest possible data for training and does not involve random sampling. A survey on loo error and results for SVM and related algorithms can be found in Elisseeff and Pontil (2002). However, loo also exhibits serious limitations. In addition to the fact that it is computationally expensive, it has a larger variance than cross validation (Hastie, Tibshirani, \& Friedman, 2002).

A way of saving the computational cost of loo is by a bound from theoretical derivation. The goal of this letter is to make radius margin bound, a theoretical bound of loo error, a practical tool. There are some interesting results on the loo bounds of SVM and kernel machines in Zhang (2001).

First, we briefly describe the SVM formulation. Given training vectors $x_{i} \in R^{n}, i=1, \ldots, l$, in two classes, and a vector $y \in R^{l}$ such that $y_{i} \in\{1,-1\}$, the SVM formulation is as follows:

$$
\begin{aligned}
\min _{w, b} & \frac{1}{2} w^{T} w \\
\text { subject to } & y_{i}\left(w^{T} \phi\left(x_{i}\right)+b\right) \geq 1, i=1, \ldots, l,
\end{aligned}
$$

where training data $x_{i}$ are mapped to a higher-dimensional space by the function $\phi$. Practically, we need only $K\left(x_{i}, x_{j}\right)=\phi\left(x_{i}\right)^{T} \phi\left(x_{j}\right)$, which is called the kernel function. In this letter, we focus on the radial basis function (RBF) kernel,

$$
K\left(x_{i}, x_{j}\right)=e^{-\left\|x_{i}-x_{j}\right\|^{2} /\left(2 \sigma^{2}\right)} .
$$

The parameter $\sigma$ can be determined by the loo bound described below. Vapnik (1998) showed that the following radius margin bound holds for SVM without the bias term $b$,

$$
\text { loo } \leq 4 R^{2}\|w\|^{2},
$$

where loo is the number of loo errors, $w$ is the solution of equation 1.1, and $R$ is the radius of the smallest sphere containing all $\phi\left(x_{i}\right)$. Vapnik and Chapelle (2000) further extend the bound for the general case where $b$ is present. It has been shown (Vapnik, 1998) that $R^{2}$ is the objective value of the following optimization problem:

$$
\begin{array}{cl}
\max _{\beta} & 1-\beta^{T} K \beta \\
\text { subject to } & 0 \leq \beta_{i}, i=1, \ldots, l, \\
& e^{T} \beta=1 .
\end{array}
$$

Some early experiments on minimizing the right-hand side of equation 1.2 are in Schölkopf, Burges, and Vapnik (1995) and Cristianini, Campbell, and Shawe-Taylor (1999). 
However, equation 1.1 is not a form for practical use. It may not be feasible if $\phi\left(x_{i}\right)$ are not linearly separable. In addition, a highly nonlinear $\phi$ may lead to overfitting. Thus, practically we solve either

$$
\min _{w, b, \xi} \frac{1}{2} w^{T} w+C \sum_{i=1}^{l} \xi_{i}
$$

or

$$
\min _{w, b, \xi} \frac{1}{2} w^{T} w+\frac{C}{2} \sum_{i=1}^{l} \xi_{i}^{2}
$$

both subject to $y_{i}\left(w^{T} \phi\left(x_{i}\right)+b\right) \geq 1-\xi_{i}$ and $\xi_{i} \geq 0$, where $\xi_{i}$ represents the training error and the parameter $C$ adjusts the training error and the regularization term $w^{T} w / 2$. We refer to the two cases as L1-SVM and L2SVM, respectively. Note that for L2-SVM we use C/2 instead of $C$ for easier analyses later. Then, if the RBF kernel is used, $C$ and $\sigma$ are the two tunable parameters. Usually they are solved through dual problems. For equation 1.4, its dual is

$$
\begin{array}{cl}
\max _{\alpha} & e^{T} \alpha-\frac{1}{2} \alpha^{T} Q \alpha \\
\text { subject to } & 0 \leq \alpha_{i} \leq C, i=1, \ldots, l, \\
& y^{T} \alpha=0
\end{array}
$$

where $e$ is the vector of all ones and $Q$ is an $l \times l$ matrix with $Q_{i j}=y_{i} y_{j} K\left(x_{i}, x_{j}\right)$. For primal and dual optimal solutions,

$$
w=\sum_{i=1}^{l} \alpha_{i} y_{i} \phi\left(x_{i}\right) \quad \text { and } \quad \frac{1}{2} w^{T} w+C \sum_{i=1}^{l} \xi_{i}=e^{T} \alpha-\frac{1}{2} \alpha^{T} Q \alpha .
$$

For equation 1.5 , its dual is

$$
\begin{array}{cl}
\max _{\alpha} & e^{T} \alpha-\frac{1}{2} \alpha^{T}\left(Q+\frac{I}{C}\right) \alpha \\
\text { subject to } & 0 \leq \alpha_{i}, i=1, \ldots, l, \\
& y^{T} \alpha=0 .
\end{array}
$$

Unfortunately, for L1-SVM, the radius margin bound, equation 1.2, does not hold, as the optimization formulation is now different. However, L2SVM can be reduced to a form of equation 1.1 using

$$
\tilde{w} \equiv\left[\begin{array}{c}
w \\
\sqrt{C} \xi
\end{array}\right] \text { and the } i \text { th training data as }\left[\begin{array}{c}
\phi\left(x_{i}\right) \\
\frac{y_{i} e_{i}}{\sqrt{C}}
\end{array}\right],
$$


where $e_{i}$ is a zero vector of length $l$ except the $i$ th component is one. The kernel function becomes $\tilde{K}\left(x_{i}, x_{j}\right)=K\left(x_{i}, x_{j}\right)+\delta_{i j} / C$, where $\delta_{i j}=1$ if $i=j$ and 0 otherwise. Then equation 1.2 can be directly used, and so existing work on the radius margin bound mainly focuses on L2-SVM (Chapelle, Vapnik, Bousquet, \& Mukherjee, 2002; Keerthi, 2002). Note that $R^{2}$ is now also different, so we will denote the new bound as

$$
\tilde{R}^{2}\|\tilde{w}\|^{2}
$$

where $\tilde{R}^{2}$ is the objective value of the following problem:

$$
\begin{aligned}
\max _{\beta} & 1+\frac{1}{C}-\beta^{T}\left(K+\frac{I}{C}\right) \beta \\
\text { subject to } & 0 \leq \beta_{i}, i=1, \ldots, l, \\
& e^{T} \beta=1 .
\end{aligned}
$$

Chapelle et al. (2002) is the first to use the differentiability of equation 1.2 and develop optimization algorithms for finding optimal $C$ and $\sigma$. This is faster than a two-dimensional grid search. More implementation issues for solving large problems are discussed in Keerthi (2002). There are several other bounds on the loo error, which may be used for model selection. Joachims (2000) developed the $\xi \alpha$-estimator, extending the results of Jaakkola and Haussler (1999) to general SVMs. Wahba, Lin, and Zhang (2000) suggested that generalized approximate cross validation (GACV) is a reasonable estimate of the generalized comparative Kullback-Liebler Distance (GCKL) (Wahba, 1998). For both $\xi \alpha$ and GACV bounds, they may not be differentiable, so efficient implementations have not been available. Vapnik and Chapelle (2000) proposed the span bound on loo, and there are also simplified approximations of the span bound. It is a very tight bound but requires more computational cost. A comparison of different methods for model selection is in Duan, Keerthi, and Poo (2003), which shows the radius margin bound for L2-SVM performs quite well. In this letter, we will discuss only radius margin bounds.

For L1-SVM, we have no way to reduce it to a form of equation 1.1. Zhang (2001) shows that it is more difficult to obtain a quantitative loo bound for nonseparable problems, which is the case for L1-SVM. Since equation 1.2 does not hold, some modifications are necessary. Duan et al. (2002), following the suggestion by Chapelle, consider

$$
D^{2} e^{T} \alpha+\sum_{i=1}^{l} \xi_{i},
$$

where $D=2 R$.

However, experiments in Duan et al. (2003) showed that comparing to other methods, this bound is not good. In addition, implementation issues such as the differentiability of equation 1.12 have not been addressed. 
In this letter, we investigate the following issues:

- Why do experiments in Duan et al. (2003) show that the radius margin bound for L2-SVM is better than that for L1-SVM? Our analysis in section 2 shows that compared to the bound for L1-SVM, the one for L2-SVM possesses some nice properties so that minima of the radius margin bound happen in the region where the generalization error is small. We show that finding a bound whose minima are in a good region may be more important than its tightness.

- Can we have better modified radius margin bounds for L1-SVM? Based on the discussion for L2-SVM, in section 3, we propose some modifications for L1-SVM that perform better than equation 1.12. However, these bounds, including equation 1.12, may not be differentiable, so gradient-based optimization methods are not applicable. We propose some further modifications for L1-SVM bounds that are differentiable.

- Should we use L1-SVM or L2-SVM after everything is considered? In section 4 , we show that in terms of generalization as measured by testing accuracy, the proposed modification of the radius margin bound for L1-SVM is competitive with that for L2-SVM. As the number of support vectors as well as free support vectors affect computational time of gradient-based methods, we also conduct comparisons. Results indicate that L1-SVM requires fewer support vectors. However, we also show that an efficient implementation for L1-SVM is not as straightforward as that for L2-SVM. In section 5, we discuss many implementation issues that were not studied before.

\section{Radius Margin Bound for L2-SVM}

In this section, we investigate why the radius margin (RM) bound performs well for L2-SVM. First, in Table 1 we list test accuracy given in Duan et al. (2003) by comparing equation 1.12, a modified RM bound for L1-SVM, and the RM bound for L2-SVM. For each problem, we fix $C$ (or $\sigma^{2}$ ) as they used and then search for the value of $\sigma^{2}$ (or $C$ ) that minimizes the bound. The $(C$, $\left.\sigma^{2}\right)$ is then used to train a model and predict the test data. Following them, we present $\ln \sigma^{2}$ and $\ln C$ using the natural $\log$. We give the description of data sets in section 4 .

Some immediate observations are as follows:

- No matter $C$ or $\sigma$ is fixed, the RM bound for L2-SVM is better.

- When $C$ is fixed, except for problem tree, the modified RM bound for L1-SVM in equation 1.12 has minima at large $\sigma$. In other words, a good $\sigma$ should be smaller.

- When $\sigma$ is fixed, for each problem, the modified RM bound for L1-SVM in equation 1.12 has the minimum at a smaller value than the best $C$. 
Table 1: Error Rates with Respect to the Test Set Obtained Using L1- and L2-SVM RM Bounds at Their Minimal Points.

\begin{tabular}{cccccc}
\hline Data Set & banana & image & splice & waveform & tree \\
& \multicolumn{5}{c}{ C fixed } \\
Value of $\ln C$ & 5.2 & 4.0 & 0.4 & 1.4 & 8.6 \\
L1 test error & $0.1043(0.6)$ & $0.0188(1.0)$ & $0.0947(3.4)$ & $0.1022(3.2)$ & $0.1086(3.8)$ \\
$D^{2}\left(e^{T} \alpha\right)+\sum \xi_{i}$ & $0.5594(10.0)$ & $0.2564(10.0)$ & $0.1545(8.1)$ & $0.1533(8.0)$ & $0.1703(-2.5)$ \\
Value of $\ln C$ & -0.9 & 0.44 & 6.91 & 0 & 9.80 \\
L2 test error & $0.1118(-1.4)$ & $0.0238(0.5)$ & $0.0947(3.3)$ & $0.0989(2.7)$ & $0.1049(4.6)$ \\
$\tilde{R}^{2}\|\tilde{w}\|^{2}$ & $0.1141(-1.6)$ & $0.0297(-0.3)$ & $0.0998(3.1)$ & $0.1030(2.1)$ & $0.1703(-2.5)$ \\
& \multicolumn{7}{c}{$\sigma$ fixed } & & \\
Value of $\ln \sigma^{2}$ & 0.6 & 1.0 & 3.4 & 3.2 & 3.8 \\
L1 test error & $0.1043(5.2)$ & $0.0188(3.9)$ & $0.0947(0.4)$ & $0.1022(1.4)$ & $0.1086(8.6)$ \\
$D^{2}\left(e^{T} \alpha\right)+\sum \xi_{i}$ & $0.3843(-2.9)$ & $0.1287(-2.9)$ & $0.1945(-2.4)$ & $0.1409(-2.5)$ & $0.2609(-10.0)$ \\
Value of $\ln \sigma^{2}$ & -1.39 & -0.29 & 3.07 & 2.80 & 4.60 \\
L2 test error & $0.1120(-0.0)$ & $0.0218(2.4)$ & $0.1007(2.1)$ & $0.0991(-0.0)$ & $0.1049(9.7)$ \\
$\tilde{R}^{2}\|\tilde{w}\|^{2}$ & $0.1124(-0.9)$ & $0.0297(0.4)$ & $0.1016(10.0)$ & $0.1007(-0.6)$ & $0.1413(-1.4)$ \\
\hline
\end{tabular}

Notes: For the case of $C$ fixed, the value given in parentheses is the value of $\ln \sigma^{2}$ where the bound attained the minimum. For the case of $\sigma$ fixed, the value given in the parentheses is the value of $\ln C$ where the bound attained the minimum.

Therefore, equation 1.12 suffers from the problem that the obtained $C$ is too small and $\sigma$ is too large. While it may not be easy to explain these phenomena directly, here, we have an alternative way of thinking: the RM bound for L2-SVM may have inherently avoided that the minimum happens at too small $C$ or too large $\sigma$. In the following, we derive two other bounds for L2-SVM and through the comparison we show that the RM bound for L2-SVM really possesses such mechanisms.

The RM bound for L2-SVM is derived using inequality 2.1 on the hardmargin SVM (see, for example, lemma 3 of Vapnik \& Chapelle, 2000). If in the loo procedure a support vector $x_{p}$ corresponding to a nonzero dual variable $\alpha_{p}>0$ is recognized incorrectly, then

$$
\frac{1}{2 \max _{i \neq j}\left\|\tilde{z}_{i}-\tilde{z}_{j}\right\|^{2}} \leq \frac{\alpha_{p}^{2}}{2} \min _{\tilde{z} \in \Lambda_{p}^{*}}\left\|\tilde{z}_{p}-\tilde{z}\right\|^{2},
$$

where

$$
\tilde{z}_{i} \equiv\left[\phi\left(x_{i}\right)^{T}, e_{i}^{T} / \sqrt{C}\right]^{T} .
$$

Equation 2.2 changes L2-SVM to a hard-margin SVM formulation and $\Lambda_{p}^{*}$ is a subset of the convex hull by $\left\{\tilde{z}_{1}, \ldots, \tilde{z}_{l}\right\} \backslash\left\{\tilde{z}_{p}\right\}$. Notice that in Vapnik and Chapelle (2000), the left-hand side of equation 2.1 is bounded below by $1 /\left(2 \tilde{D}^{2}\right)$. Checking the derivation, we realize that the proof of lemma 3 in Vapnik and Chapelle (2000) remains valid when substituting $1 /\left(2 \tilde{D}^{2}\right)$ with a larger number $1 /\left(2 \max _{i \neq j}\left\|\tilde{z}_{i}-\tilde{z}_{j}\right\|^{2}\right)$, and the inequality still holds. 
By the definition of $\Lambda_{p}^{*}$,

$$
\min _{\tilde{z} \in \Lambda_{p}^{*}}\left\|\tilde{z}_{p}-\tilde{z}\right\|^{2} \leq \max _{i \neq j}\left\|\tilde{z}_{i}-\tilde{z}_{j}\right\|^{2} .
$$

Since $\tilde{D}$ is the diameter of the smallest sphere containing all $\tilde{z}_{1}, \ldots, \tilde{z}_{l}$,

$$
\max _{i \neq j}\left\|\tilde{z}_{i}-\tilde{z}_{j}\right\|^{2} \leq \tilde{D}^{2}=4 \tilde{R}^{2} .
$$

Therefore, if $x_{p}$ is recognized incorrectly, equations 2.1, 2.3, and 2.4 imply $\alpha_{p}^{2} \tilde{D}^{4} \geq 1$. Then $\alpha_{p} \tilde{D}^{2} \geq 1$, so with $\sum_{p} \alpha_{p}=\|\tilde{w}\|^{2}$, we have

$$
\text { loo } \leq \sum_{p=1}^{l} \alpha_{p} \tilde{D}^{2}=4\|\tilde{w}\|^{2} \tilde{R}^{2} .
$$

Instead of using equation 2.4, from equation 2.2, we consider

$$
\left\|\tilde{z}_{i}-\tilde{z}_{j}\right\|^{2}=\frac{2}{C}+\left\|\phi\left(x_{i}\right)-\phi\left(x_{j}\right)\right\|^{2}
$$

where $i \neq j$. With

$$
\left\|\phi\left(x_{i}\right)-\phi\left(x_{j}\right)\right\|^{2} \leq D^{2}=4 R^{2},
$$

where $R^{2}$ is the objective value of equation 1.3 , we obtain a different bound:

$$
l o o \leq\left(4 R^{2}+\frac{2}{C}\right)\|\tilde{w}\|^{2} .
$$

Some immediate comparisons between the two bounds are as follows. When $\sigma^{2}$ is fixed, we can easily prove that

$$
\lim _{C \rightarrow \infty} \tilde{R}^{2}=\lim _{C \rightarrow \infty} R^{2}=\lim _{C \rightarrow \infty}\left(R^{2}+\frac{0.5}{C}\right) .
$$

Furthermore, we have the following:

Theorem 1. With fixed $\sigma^{2}$,

$$
\left(R^{2}+\frac{0.5}{C}\right)\|\tilde{w}\|^{2} \leq \tilde{R}^{2}\|\tilde{w}\|^{2},
$$

and $\tilde{R}^{2} /\left(R^{2}+\frac{0.5}{C}\right)$ is a monotonically decreasing function of $C$.

Thus, equation 2.5 is a tighter bound. The proof is in appendix A. 
When $C$ is small, a large $1 / C$ causes $\tilde{R}^{2}$ to be dominated by the term $1 / C-\beta^{T} \beta / C$ in the objective function of equation 1.11. If we consider $\beta_{i}=$ $1 / l, i=1, \ldots, l$, a feasible solution of equation 1.11 , then with $1-\beta^{T} K \beta \geq 0$,

$$
1+\frac{1}{C} \geq \tilde{R}^{2} \geq 1+\frac{1}{C}-\beta^{T}\left(K+\frac{I}{C}\right) \beta \geq \frac{1}{C}\left(1-\frac{1}{l}\right) .
$$

Thus, if $l \gg 1$ and $C$ is small,

$$
\tilde{R}^{2} \approx \frac{1}{C}
$$

but

$$
\left(R^{2}+\frac{0.5}{C}\right) \approx \frac{0.5}{C}
$$

Therefore, when $C$ is small, equation 1.10 overestimates the loo error. Interestingly this becomes a good property due to the following reason. As we can see in equation 2.1, the RM bound seriously overestimates the loo if $\alpha_{p}$ is large. This happens only when $C$ is not small. It is very easy to see this for L1-SVM due to the constraint $\alpha_{i} \leq C$. For L2-SVM, when $C$ is small, we can show that $\alpha_{i} \rightarrow \frac{l_{2}}{l_{1}} C$ if $y_{i}=1$ and $\alpha_{i} \rightarrow \frac{l_{1}}{l_{2}} C$ if $y_{i}=-1$, where $l_{1}$ and $l_{2}$ are numbers of data with $y_{i}=1$ and -1 , respectively. The derivation is similar to equation 2.7. Thus, large $\alpha$ happens only when $C$ is large. Therefore, the overestimation of loo at large $C$ pushes the minimum to be at a smaller value of $C$. Therefore, we can see that $\tilde{R}^{2}$ puts the penalty at small $C$ so the minimum of the RM bound may be pushed back to the correct position. In Table 2, we will see that when $\sigma$ is fixed, $\left(R^{2}+\frac{0.5}{C}\right)\|\tilde{w}\|^{2}$ always returns a smaller $C$ than $\tilde{R}^{2}\|\tilde{w}\|^{2}$.

Next, we present another bound for L2-SVM. If the problem is separable (i.e., hard-margin SVM) and the set of support vectors remains the same during the leave-one-out procedure, theorem 3 of Vapnik and Chapelle (2000) shows

$$
y_{p}\left(f^{0}\left(x_{p}\right)-f^{p}\left(x_{p}\right)\right)=\alpha_{p}^{0} s_{p}^{2},
$$

where $x_{p}$ is any support vector, $\alpha_{p}^{0}$ is the $p$ th element of the dual solution $\alpha^{0}$ by training the whole set, $f^{0}$ and $f^{p}$ are the decision functions trained, respectively, on the whole set and after the point $x_{p}$ has been removed, and

$$
S_{p}^{2} \equiv \min \left\{\left(\sum_{i=1}^{l} \lambda_{i} \phi\left(x_{i}\right)\right)^{2} \mid \lambda_{p}=-1, \sum_{i=1}^{l} \lambda_{i}=0, \alpha_{i}^{0}+y_{i} y_{p} \alpha_{p}^{0} \lambda_{i} \geq 0\right\} .
$$

Note that the assumption that support vectors do not change might not always be true so we must be cautious that things derived from equation 2.9 might not be real bounds of loo. 
Table 2: Error Rates with Respect to the Test Set Obtained Using Three L2-SVM RM Bounds.

\begin{tabular}{|c|c|c|c|c|c|}
\hline Data Set & banana & image & splice & waveform & tree \\
\hline \multicolumn{6}{|c|}{$C$ fixed } \\
\hline Value of $\ln C$ & -0.9 & 0.44 & 6.91 & 0 & 9.80 \\
\hline L2 test & $0.1118(-1.4)$ & $0.0238(0.5)$ & $0.0947(3.3)$ & $0.0989(2.7)$ & $0.1049(4.6)$ \\
\hline$\tilde{R}^{2}\|\tilde{w}\|^{2}$ & $0.1141(-1.6)$ & $0.0297(-0.3)$ & $0.0998(3.1)$ & $0.1030(2.1)$ & $0.1703(-2.5)$ \\
\hline$\left(R^{2}+0.5 / C\right)\|\tilde{w}\|^{2}$ & $0.1141(-1.6)$ & $0.0297(-0.3)$ & $0.0998(3.1)$ & $0.1267(5.6)$ & $0.1703(-2.5)$ \\
\hline$\left(R^{2}+0.25 / C\right)\|\tilde{w}\|^{2}$ & $0.1141(-1.6)$ & $0.2723(9.5)$ & 0.0998(3.1) & $0.1354(6.5)$ & $0.1703(-2.5)$ \\
\hline \multicolumn{6}{|c|}{$\sigma$ fixed } \\
\hline Value of $\ln \sigma^{2}$ & -1.39 & -0.29 & 3.07 & 2.80 & 4.60 \\
\hline L2 test error & $0.1120(-0.0)$ & $0.0218(2.4)$ & $0.1007(2.1)$ & $0.0991(-0.0)$ & $0.1049(9.7)$ \\
\hline$\tilde{R}^{2}\|\tilde{w}\|^{2}$ & $0.1124(-0.9)$ & $0.0297(0.4)$ & $0.1016(10.0)$ & $0.1007(-0.6)$ & $0.1413(-1.4)$ \\
\hline$\left(R^{2}+0.5 / C\right)\|\tilde{w}\|^{2}$ & $0.1145(-1.5)$ & $0.0366(-0.7)$ & $0.1251(-1.4)$ & $0.1048(-1.4)$ & $0.1763(-2.3)$ \\
\hline$\left(R^{2}+0.25 / C\right)\|\tilde{w}\|^{2}$ & $0.1178(-2.1)$ & $0.0564(-1.8)$ & $0.4800(-10.0)$ & $0.1124(-2.2)$ & $0.2609(-3.1)$ \\
\hline
\end{tabular}

Notes: For the case of $C$ fixed, the value given in parentheses is the value of $\ln \sigma^{2}$ where the bound attained the minimum. For the case of $\sigma$ fixed, the value given in the parentheses is the value of $\ln C$ where the bound attained the minimum.

For L2-SVM, by equation 1.9 , it can be reduced to the hard-margin form. Equation 2.9 becomes

$$
y_{p}\left(\tilde{f}^{0}\left(\tilde{z}_{p}\right)-\tilde{f}^{p}\left(\tilde{z}_{p}\right)\right)=\alpha_{p}^{0} \tilde{S}_{p}^{2}
$$

where $\tilde{z}_{p}$ is defined in equation 2.2. We then have the following lemma:

Lemma 1. If the set of support vectors remains the same during the leave-one-out procedure, we have

$$
\tilde{S_{p}^{2}} \leq D^{2}+\frac{1}{C}+ \begin{cases}\frac{1}{\left(l_{s v 1}-1\right) C} & \text { if } y_{p}=1, \\ \frac{1}{\left(l_{s v 2}-1\right) C} & \text { if } y_{p}=-1,\end{cases}
$$

where $l_{s v 1}$ and $l_{s v 2}$ are the number of support vectors with $y_{i}=1$ and -1 , respectively.

The proof is in appendix B. When there is an loo error, $y_{p} \tilde{f}^{p}\left(\tilde{z}_{p}\right) \leq 0$ and $y_{p} \tilde{f}^{0}\left(\tilde{z}_{p}\right)=1$. Thus, if $y_{p}=1$ and $\alpha_{p}^{0}<C$,

$$
\begin{aligned}
1 & \leq y_{p}\left(\tilde{f}^{0}\left(\tilde{z}_{p}\right)-\tilde{f}^{p}\left(\tilde{z}_{p}\right)\right) \\
& =\alpha_{p}^{0} \tilde{S}_{p}^{2} \\
& \leq \alpha_{p}^{0}\left(D^{2}+\frac{1}{C}+\frac{1}{\left(l_{s v 1}-1\right) C}\right) .
\end{aligned}
$$


Similarly, if $y_{p}=-1$ and $\alpha_{p}^{0}<C$, then

$$
1 \leq \alpha_{p}^{0}\left(D^{2}+\frac{1}{C}+\frac{1}{\left(l_{s v 2}-1\right) C}\right) .
$$

It is trivial that

$$
1 \leq \alpha_{p}^{0}\left(D^{2}+\frac{1}{C}\right)
$$

if $\alpha_{p}^{0} \geq C$.

Therefore, from equation 2.10 to 2.12 , we have

$$
\begin{aligned}
l o o & \leq \sum_{p=1}^{l} \alpha_{p}\left(D^{2}+\frac{1}{C}\right)+\sum_{y_{p}=1, \alpha_{p}^{0}<C} \frac{\alpha_{p}^{0}}{\left(l_{s v 1}-1\right) C}+\sum_{y_{p}=-1, \alpha_{p}^{0}<C} \frac{\alpha_{p}^{0}}{\left(l_{s v 2}-1\right) C} \\
& \leq \sum_{p=1}^{l} \alpha_{p}^{0}\left(D^{2}+\frac{1}{C}\right)+\frac{l_{s v 1}}{l_{s v 1}-1}+\frac{l_{s v 2}}{l_{s v 2}-1} .
\end{aligned}
$$

Since $l_{s v 1} /\left(l_{s v 1}-1\right)$ and $l_{s v 2} /\left(l_{s v 2}-1\right)$ are constants, $e^{T} \alpha\left(D^{2}+1 / C\right)$ can be considered as a bound of loo for L2-SVM. With $\|\tilde{w}\|^{2}=e^{T} \alpha$ for L2-SVM, a new bound is

$$
\left(R^{2}+\frac{0.25}{C}\right)\|\tilde{w}\|^{2}
$$

Note that using $\alpha=C \xi$ for L2-SVM, this bound can be rewritten as

$$
D^{2} e^{T} \alpha+\sum_{i=1}^{l} \xi_{i},
$$

the same form as equation 1.12 for L1-SVM.

Interestingly, it is an even tighter bound than equation 2.5 as $R^{2}+\frac{0.25}{C}$ is smaller than $R^{2}+\frac{0.5}{C}$. In Table 2 we present results using the two new bounds for L2-SVM. It can be clearly seen that both new bounds perform more poorly than the original RM bound, and equation 2.13 is particularly bad. Following are some further observations:

- When $C$ is fixed, the accuracy of using equation 2.5 for waveform is not good because it obtains too large a $\sigma$. For equation 2.13, it returns an even larger $\sigma$, so the error rate increases further. Especially for the problem image, a very large $\ln \sigma^{2}=9.5$ is obtained. 
If we check the limiting behavior of these three bounds, when $\sigma \rightarrow \infty$,

$$
\begin{aligned}
& \tilde{R}^{2}\|\tilde{w}\|^{2} \approx \frac{4 l_{1} l_{2}}{l}, \\
& \left(R^{2}+\frac{0.5}{C}\right)\|\tilde{w}\|^{2} \approx \frac{2 l_{1} l_{2}}{l}, \\
& \left(R^{2}+\frac{0.25}{C}\right)\|\tilde{w}\|^{2} \approx \frac{l_{1} l_{2}}{l},
\end{aligned}
$$

where $l_{1}$ and $l_{2}$ are the number of data with $y_{i}=1$ and -1 , respectively. We leave the details of equations 2.15 through 2.17 to appendix $C$.

Therefore, we suspect that smaller values when $\sigma \rightarrow \infty$ cause the bound 2.13 to have minima at large $\sigma$. To confirm this, in Figure 1, we present the value of equation 2.13 , using the problem image.

Clearly there are two local minima where the left one is better but is not chosen. This seems to suggest that as equation 1.10 has larger values when $\sigma \rightarrow \infty$, it can avoid that the global minimum happens at a wrong place.

- When $\sigma^{2}$ is fixed, the situation is also similar, as is shown in Figure 1. The tighter the bound is, the worse the test accuracy is as the optimal $C$ becomes too small. If $R^{2}>1 / 2$, we can further prove that changing the bound from $\tilde{R}^{2}$ to $R^{2}+\frac{0.5}{C}$ and then $R^{2}+\frac{0.25}{C}$, the optimal $C$ can only be smaller. In the proof of theorem 1 , we show that $R^{2} \geq 1 / 2$ and then $\tilde{R}^{2} /\left(R^{2}+\frac{0.5}{C}\right)$ is a decreasing function of $C$. If we further assume $R^{2}>1 / 2$, both $\tilde{R}^{2} /\left(R^{2}+\frac{0.5}{C}\right)$ and $\left(R^{2}+\frac{0.5}{C}\right) /\left(R^{2}+\frac{0.25}{C}\right)$ are strictly decreasing functions of $C$ so this result on the position of optimal $C$ follows from the following theorem (the proof is in appendix E):

Theorem 2. If $f_{1}, f_{2}, g>0$ are positive functions on $(0, \infty)$ and $f_{1} / f_{2}$ is strictly decreasing, then any $f_{2} g^{\prime} s$ global optimum is greater than or equals that of $f_{1} g$.

Therefore, as we have mentioned earlier, there is a benign overestimation for $\tilde{R}$ when $C$ is small. In summary, the experiments in this section tell us that:

- Finding a bound whose minima are in a good region may be more important than its tightness.

- A good bound should avoid minima that happen at the boundary (i.e., too small or too large $C$ and $\sigma^{2}$ ).

\section{Some Heuristic Bounds for L1-SVM}

Based on the experience in the previous section, we search for better radius margin bounds for L1-SVM that can replace the $D^{2} e^{T} \alpha+\sum_{i=1}^{l} \xi_{i}$ used in 

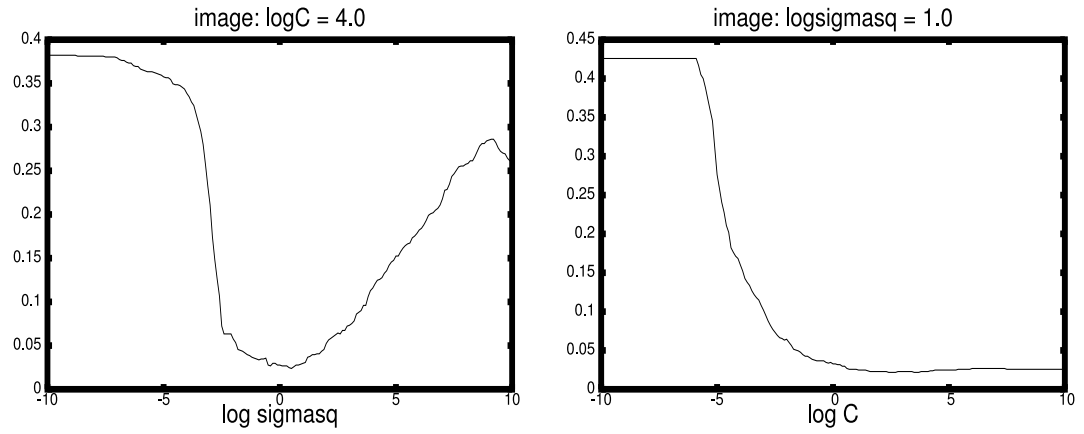

Testing error
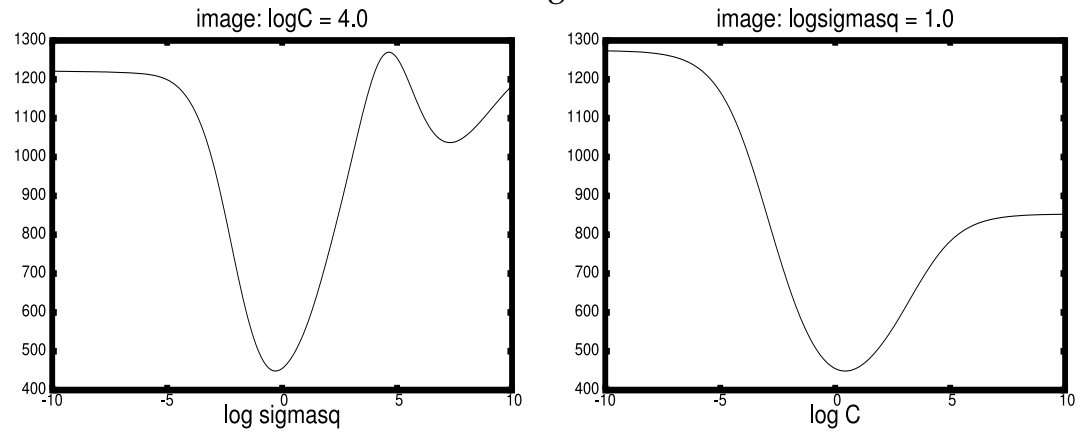

The RM bound for L2-SVM
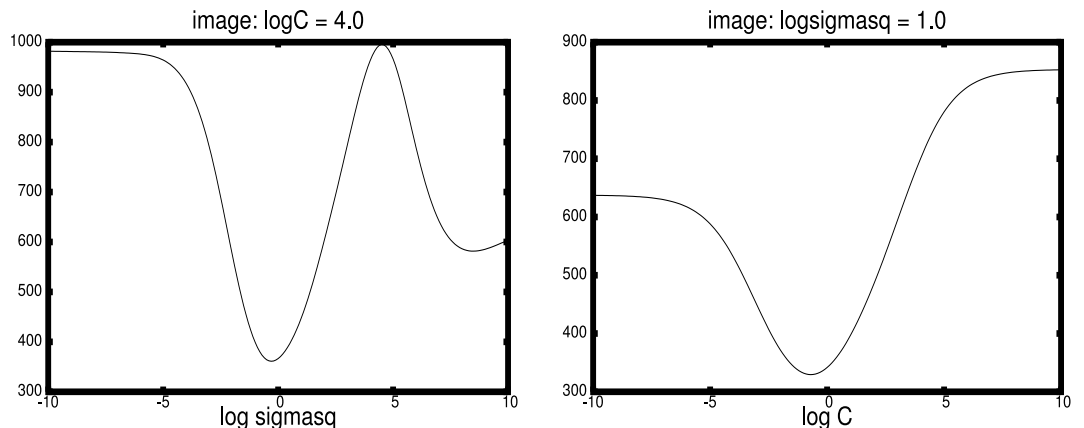

The bound $\left(R^{2}+0.5 / C\right)\|\tilde{w}\|^{2}$

image: $\log C=4.0$

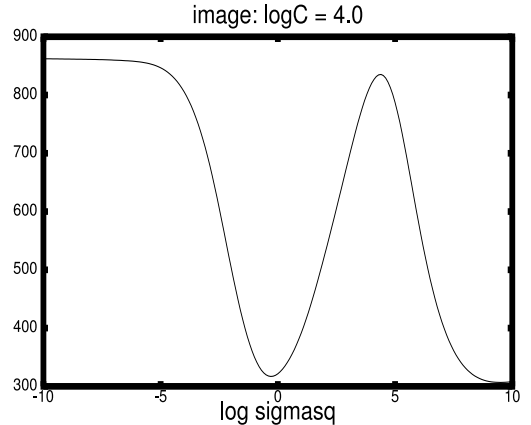

image: logsigmasq $=1.0$

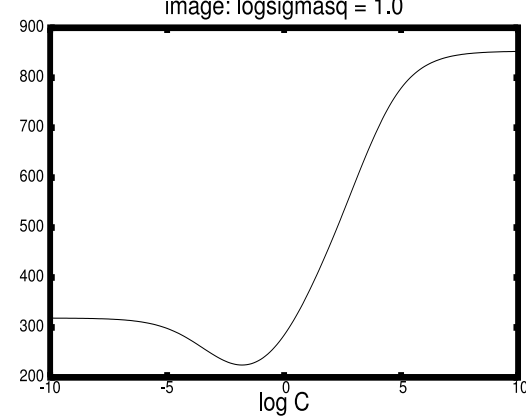

The bound $\left(R^{2}+0.25 / C\right)\|\tilde{w}\|^{2}$ 
Duan et al. (2003). Our strategy is to consider that equation 2.13 for L2SVM is the counterpart of $D^{2} e^{T} \alpha+\sum_{i=1}^{l} \xi_{i}$ for L1-SVM, as the explanation in equation 2.14 shows they have the same form. Then, by investigating the difference between equations 1.10 and 2.13, we seek the counterpart of equation 1.10 for L1-SVM.

If we consider

$$
\tilde{R}^{2} \approx R^{2}+\frac{1}{C}
$$

$\tilde{R}^{2}\|\tilde{w}\|^{2}$ is similar to

$$
\left(R^{2}+\frac{1}{C}\right)\|\tilde{w}\|^{2}=R^{2} e^{T} \alpha+\sum_{i=1}^{l} \xi_{i}
$$

as $\|\tilde{w}\|^{2}=e^{T} \alpha$ and $\alpha=C \xi$ for L2-SVM.

Thus, for L1-SVM,

$$
R^{2} e^{T} \alpha+\sum_{i=1}^{l} \xi_{i}
$$

may be a good bound. Now, using equation 1.7,

$$
C \sum_{i=1}^{l} \xi_{i}=e^{T} \alpha-\|w\|^{2} \leq e^{T} \alpha
$$

so another possibility is

$$
\left(R^{2}+\frac{1}{C}\right) e^{T} \alpha=\left(R^{2}+\frac{1}{C}\right)\left(\|w\|^{2}+C \sum_{i=1}^{l} \xi_{i}\right)
$$

Later, we will conduct experiments on these two new bounds. Another issue about these bounds is their differentiability. We would like them to be differentiable so gradient-based methods can be used to find a local minimum. Unfortunately, as we show in appendix F, the two new bounds for L1-SVM may not be differentiable.

Figure 1: Facing page. image data set. The figures on the left-hand side show the values of the bounds with respect to $\ln \sigma^{2}$ with fixed $\ln C$. The figures on the right-hand side show the values of the bounds with respect to $\ln C$ with fixed $\ln \sigma^{2}$. For the case of $\ln \sigma^{2}$ fixed, we can see that the local minimum of larger $C$ becomes dominant as the bound becomes tighter. For the case of $\ln C$ fixed, the tighter the bound is, the smaller the optimal $C$ is. 
Therefore, if we require differentiability at any $C$ and $\sigma^{2}$, the above two new bounds for L1-SVM are not applicable. Thus, we propose a further modification of equation 3.2,

$$
\left(R^{2}+\frac{\Delta}{C}\right)\left(\|w\|^{2}+2 C \sum_{i=1}^{l} \xi_{i}\right),
$$

where $\Delta$ is a positive constant close to one. As we have discussed, $\Delta / C$ can be thought of as a penalty term for small $C$. If we take $\Delta=1$, the main change from equation 3.2 is to replace $e^{T} \alpha$ by a differentiable term.

This modified bound is still an loo bound. Moreover, it does not need the assumption that support vectors do not change. From lemma 3 of Vapnik and Chapelle (2000) and $S_{p} \leq D$, we have

$$
\begin{aligned}
1 & \leq \alpha_{p}^{0} S_{p} \max \left(D, \frac{1}{\sqrt{C}}\right) \\
& \leq \alpha_{p}^{0} \max \left(D, \frac{1}{\sqrt{C}}\right)^{2} \\
& \leq \alpha_{p}^{0}\left(D^{2}+\frac{1}{C}\right)
\end{aligned}
$$

where $\alpha_{p}^{0}$ is the $p$ th element of the dual solution $\alpha^{0}$ by training the whole set. Therefore,

$$
\begin{aligned}
\text { loo } & \leq \sum_{p=1}^{l} \alpha_{p}^{0}\left(D^{2}+\frac{1}{C}\right) \\
& =\left(\|w\|^{2}+C \sum_{i=1}^{l} \xi_{i}\right)\left(D^{2}+\frac{1}{C}\right) \\
& \leq\left(\|w\|^{2}+2 C \sum_{i=1}^{l} \xi_{i}\right)\left(D^{2}+\frac{4 \Delta}{C}\right)
\end{aligned}
$$

when $\Delta \geq 1 / 4$.

Table 3 presents a comparison of different bounds for L1-SVM: equation 1.12; two improvements, equations 3.1 and 3.2; and the differentiable bound, equation 3.3 , with $\Delta=1$ and $\Delta=0.5$. It can be clearly seen that bounds proposed in this section are better than equation 1.12. Besides the differentiability, the derivative of equation 3.3 can be easily computed. Details are in section 5.1.

\section{Overall Performance of Bounds for L1-SVM and L2-SVM}

In previous sections, we have discussed some bounds for L1- and L2-SVMs. However, our experiments were based on fixing one parameter and testing 


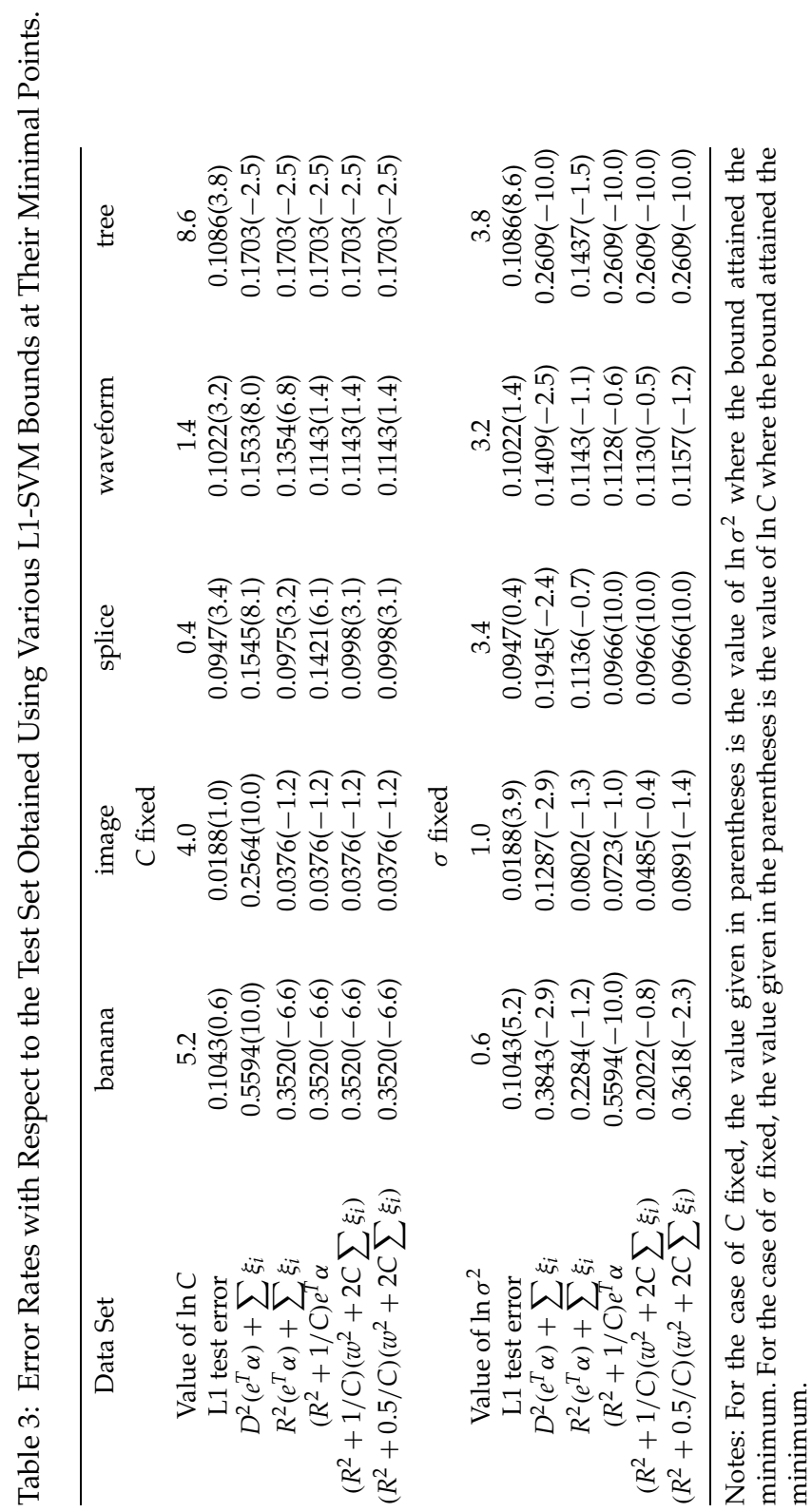


Table 4: Some Statistics About the Data Sets.

\begin{tabular}{crrrr}
\hline Data Set & banana & diabetes & image & ringnorm \\
Number of features & 2 & 8 & 18 & 20 \\
Number of training samples & 400 & 468 & 1300 & 400 \\
Number of testing samples & 4900 & 300 & 1010 & 7000 \\
Number of positive samples in training set & 183 & 170 & 560 & 207 \\
Number of positive samples in test set & 2741 & 98 & 430 & 3457 \\
\hline Data Set & splice & tree & twonorm & waveform \\
Number of features & 60 & 18 & 20 & 21 \\
Number of training samples & 1000 & 700 & 400 & 400 \\
Number of testing samples & 2175 & 11692 & 7000 & 4600 \\
Number of positive samples in training set & 517 & 502 & 207 & 268 \\
Number of positive samples in test set & 1131 & 8642 & 3496 & 3085 \\
\hline
\end{tabular}

the other one. This may not reveal how bounds perform when $C$ and $\sigma$ are considered together. In this section, we obtain the minimal point of each bound in the $\left(\ln C, \ln \sigma^{2}\right)$ plane. Then a model based on this $(C, \sigma)$ is trained to predict the test data.

In addition to the five data sets used in the previous sections, there are three more data sets here. Most of them are from Rätsch (1999), as in Duan et al. (2003). The tree data set was originally used in Bailey, Pettit, Borochoff, Manry, and Jiang (1993). Table 4 shows some statistics about the data sets.

We consider the bounded region that $\ln C$ and $\ln \sigma^{2}$ are both from -10 to 10. For L1-SVM, our new bounds tend to increase the $C$ value at the minimal point due to the change from $R^{2}$ to $R^{2}+\frac{\Delta}{C}$. Sometimes it pushes the minimal point to the boundary at $\ln C=10$. However, we observe that at a smaller $C$, with the same $\sigma$, the model already generated has no bounded support vectors. That is, $\xi_{i}=0, i=1, \ldots, l$, so the training data are fully separated. Therefore, the model using larger $C$ will be the same, and there is no reason to increase $C$ further even though the bound can still be decreased. This can be seen in Table 6 described later. In our experiment, once at a $(C, \sigma)$ if data are fully separated, for the same $\sigma$, larger $C$ will not be considered.

Table 5 presents the results of using different bounds. It shows in each block the optimal parameters $\left(\ln C, \ln \sigma^{2}\right)$ and the error rate with respect to the test set for each bound and data set. Here, we use $\Delta=1$ and $\Delta=0.5$ for our differentiable modifications of the L1-SVM bound (see equation 3.3). We discretize $[-10,10]$ to 21 points so at most 441 parameters are tried. Some observations are as follows:

- Compared with the results in section 2, the difference in performance among different bounds becomes less significant when $C$ and $\sigma$ are considered together. This shows that fixing one parameter may not be adequate all the time. 
Table 5: Error Rates with Respect to the Test Set Obtained Using Various L1and L2-SVM Bounds at Their Minimal Points.

\begin{tabular}{ccccc}
\hline Data Set & banana & diabetes & image & ringnorm \\
L1 test error & $(7,1) 0.1084$ & $(6,6) 0.2267$ & $(4,1) 0.0188$ & $(-2,2) 0.0143$ \\
L1 $D^{2} e^{T} \alpha+\sum \xi_{i}$ & $(-3,-1) 0.1482$ & $(-10,10) 0.3267$ & $(-3,1) 0.1297$ & $(-2,2) 0.0143$ \\
L1 $R^{2} e^{T} \alpha+\sum \xi_{i}$ & $(-2,-2) 0.1198$ & $(-2,2) 0.2467$ & $(-1,0) 0.0535$ & $(-1,2) 0.0161$ \\
L1 $\left(R^{2}+1 / C\right) e^{T} \alpha$ & $(-1,-1) 0.1182$ & $(-10,10) 0.3267$ & $(4,-1) 0.0356$ & $(1,2) 0.0199$ \\
L1 $\left(R^{2}+1 / C\right)\left(\|w\|^{2}+2 C \sum \xi_{i}\right)$ & $(-1,-2) 0.1135$ & $(2,-1) 0.3233$ & $(4,-1) 0.0356$ & $(1,2) 0.0199$ \\
L1 $\left(R^{2}+0.5 / C\right)\left(\|w\|^{2}+2 C \sum \xi_{i}\right)$ & $(-1,-2) 0.1135$ & $(-10,10) 0.3267$ & $(4,-1) 0.0356$ & $(1,2) 0.0199$ \\
L2 test error & $(0,-1) 0.1112$ & $(6,4) 0.2233$ & $(9,4) 0.0188$ & $(-3,2) 0.0139$ \\
L2 RM bound & $(-1,-2) 0.1141$ & $(-2,2) 0.2400$ & $(0,0) 0.0297$ & $(2,2) 0.0190$ \\
L2 $\left(R^{2}+0.5 / C\right)\|\tilde{w}\|^{2}$ & $(-2,-1) 0.1212$ & $(-3,2) 0.2367$ & $(-1,0) 0.0406$ & $(0,2) 0.0161$ \\
L2 $\left(R^{2}+0.25 / C\right)\|\tilde{w}\|^{2}$ & $(-2,-1) 0.1212$ & $(-3,2) 0.2367$ & $(-2,1) 0.0861$ & $(-1,2) 0.0147$ \\
\hline Data Set & splice & tree & twonorm & waveform \\
L1 test error & $(1,4) 0.0970$ & $(9,4) 0.1089$ & $(-2,3) 0.0234$ & $(1,3) 0.1035$ \\
L1 $D^{2} e^{T} \alpha+\sum \xi_{i}$ & $(-2,5) 0.1480$ & $(-3,1) 0.1483$ & $(-2,4) 0.0243$ & $(-2,3) 0.1322$ \\
L1 $R^{2} e^{T} \alpha+\sum \xi_{i}$ & $(-1,4) 0.1200$ & $(-2,0) 0.1378$ & $(-1,3) 0.0240$ & $(-1,2) 0.1096$ \\
L1 $\left(R^{2}+1 / C\right) e^{T} \alpha$ & $(2,3) 0.1007$ & $(-2,1) 0.1401$ & $(2,2) 0.0349$ & $(-1,3) 0.1111$ \\
L1 $\left(R^{2}+1 / C\right)\left(\|w\|^{2}+2 C \sum \xi_{i}\right)$ & $(2,3) 0.1007$ & $(3,-2) 0.1497$ & $(2,2) 0.0349$ & $(3,2) 0.1098$ \\
L1 $\left(R^{2}+0.5 / C\right)\left(\|w\|^{2}+2 C \sum \xi_{i}\right)$ & $(2,3) 0.1007$ & $(-2,1) 0.1401$ & $(2,2) 0.0349$ & $(-1,3) 0.1111$ \\
L2 test error & $(1,4) 0.0979$ & $(10,5) 0.1066$ & $(-2,2) 0.0223$ & $(0,3) 0.1011$ \\
L2 RM bound & $(10,3) 0.1007$ & $(-2,1) 0.1354$ & $(0,3) 0.0260$ & $(0,2) 0.1039$ \\
L2 $\left(R^{2}+0.5 / C\right)\|\tilde{w}\|^{2}$ & $(-1,5) 0.1292$ & $(-2,1) 0.1354$ & $(0,4) 0.0251$ & $(-1,3) 0.1041$ \\
L2 $\left(R^{2}+0.25 / C\right)\|\tilde{w}\|^{2}$ & $(-2,5) 0.1338$ & $(-3,1) 0.1433$ & $(-1,4) 0.0240$ & $(-2,3) 0.1139$ \\
\hline The & & &
\end{tabular}

Note: The value given in parentheses is the value of $\left(\ln C, \ln \sigma^{2}\right)$ where the bound attained the minimum.

- The modified RM bound $D^{2} e^{T} \alpha+\sum \xi_{i}$ performs worse than our modifications for many data sets. Similarly, its counterpart for L2-SVM, $\left(R^{2}+\frac{0.25}{C}\right)\|\tilde{w}\|^{2}$, is also the worst except for diabetes, ringnorm, and twonorm.

- Our new bounds $R^{2} e^{T} \alpha+\sum \xi_{i}$ and $\left(R^{2}+\frac{1}{C}\right) e^{T} \alpha$ for L1-SVM have generated results competitive with those by the RM bound for L2SVM.

- Since the L1-SVM counterparts of $\tilde{R}^{2}\|\tilde{w}\|^{2}$ for L2-SVM are not differentiable, we further modify them to $\left(R^{2}+\frac{1}{C}\right)\left(\|w\|^{2}+2 C \sum \xi_{i}\right)$ or $\left(R^{2}+\frac{0.5}{C}\right)\left(\|w\|^{2}+2 C \sum \xi_{i}\right)$. Results show that fortunately, the test accuracy does not change much.

Next, we compare the number of support vectors for optimal models generated by these bounds. In particular, we are interested in the difference between L1- and L2-SVM. If L1-SVM does not possess an advantage in this aspect, it may be better to consider L2-SVM as its RM bound is derived in a more natural way.

Table 6 presents the total number of support vectors and the number of free support vectors for the optimal models. There are some points to note: 
Table 6: Number of Support Vectors and Number of Free Support Vectors Obtained Using Various L1- and L2-SVM Bounds at Their Minimal Points.

\begin{tabular}{ccccc}
\hline Data Set & banana & diabetes & image & ringnorm \\
L1 test error & $(88,16)$ & $(239,18)$ & $(276,245)$ & $(212,45)$ \\
L1 $D^{2} e^{T} \alpha+\sum \xi_{i}$ & $(345,6)$ & $(340,0)$ & $(895,29)$ & $(212,45)$ \\
L1 $R^{2} e^{T} \alpha+\sum \xi_{i}$ & $(262,21)$ & $(326,12)$ & $(622,215)$ & $(145,63)$ \\
L1 $\left(R^{2}+1 / C\right) e^{T} \alpha$ & $(175,21)$ & $(340,0)$ & $(708,708)$ & $(101,101)$ \\
L1 $\left(R^{2}+1 / C\right)\left(\|w\|^{2}+2 C \sum \xi_{i}\right)$ & $(185,35)$ & $(435,435)$ & $(708,708)$ & $(101,101)$ \\
L1 $\left(R^{2}+0.5 / C\right)\left(\|w\|^{2}+2 C \sum \xi_{i}\right)$ & $(185,35)$ & $(340,0)$ & $(708,708)$ & $(101,101)$ \\
L2 test error & $(254,254)$ & $(356,356)$ & $(225,225)$ & $(400,400)$ \\
L2 RM bound & $(378,378)$ & $(447,447)$ & $(886,886)$ & $(120,120)$ \\
L2 $\left(R^{2}+0.5 / C\right)\|\tilde{w}\|^{2}$ & $(382,382)$ & $(468,468)$ & $(1120,1120)$ & $(188,188)$ \\
L2 $\left(R^{2}+0.25 / C\right)\|\tilde{w}\|^{2}$ & $(382,382)$ & $(468,468)$ & $(1182,1182)$ & $(269,269)$ \\
\hline Data Set & splice & tree & twonorm & waveform \\
L1 test error & $(601,481)$ & $(197,62)$ & $(201,16)$ & $(120,52)$ \\
L1 $D^{2} e^{T} \alpha+\sum \xi_{i}$ & $(909,13)$ & $(401,59)$ & $(242,18)$ & $(226,12)$ \\
L1 $R^{2} e^{T} \alpha+\sum \xi_{i}$ & $(728,130)$ & $(443,180)$ & $(136,32)$ & $(203,55)$ \\
L1 $\left(R^{2}+1 / C\right) e^{T} \alpha$ & $(877,877)$ & $(345,67)$ & $(159,159)$ & $(181,21)$ \\
L1 $\left(R^{2}+1 / C\right)\left(\|w\|^{2}+2 C \sum \xi_{i}\right)$ & $(877,877)$ & $(663,663)$ & $(159,159)$ & $(151,151)$ \\
L1 $\left(R^{2}+0.5 / C\right)\left(\|w\|^{2}+2 C \sum \xi_{i}\right)$ & $(877,877)$ & $(345,67)$ & $(159,159)$ & $(181,21)$ \\
L2 test error & $(761,761)$ & $(290,290)$ & $(380,380)$ & $(198,198)$ \\
L2 RM bound & $(892,892)$ & $(671,671)$ & $(179,179)$ & $(238,238)$ \\
L2 $\left(R^{2}+0.5 / C\right)\|\tilde{w}\|^{2}$ & $(956,956)$ & $(671,671)$ & $(191,191)$ & $(240,240)$ \\
L2 $\left(R^{2}+0.25 / C\right)\|\tilde{w}\|^{2}$ & $(998,998)$ & $(700,700)$ & $(286,286)$ & $(297,297)$ \\
\hline
\end{tabular}

Notes: For the L2 cases, the number of free support vectors equals the number of support vectors because there is no upper bound for $\alpha$. For the L1 cases of which the number of free support vectors equals the number of support vectors, the training data are fully separated.

- There are fewer support vectors for L1-SVM than for L2-SVM. This is consistent with the common understanding that the quadratic cost function leads to more data with small nonzero $\xi_{i}$.

- We list the number of free support vectors because they affect the training time. When we use the shrinking technique in Joachims (1998), we select the working set from the free support vectors, and thus fewer support vectors generally yields shorter training time.

- For L2-SVM, the number of free support vectors equals the number of support vectors because there is no upper bound for $\alpha$. For some problems in L1, the number of free support vectors also equals the number of support vectors because the training data are fully separated in the model generated. 


\section{Efficient Implementation by Gradient Methods}

Experiments in the previous section have shown the viability of some possible bounds for L1-SVM. In this section, we discuss their efficient implementations by numerical optimization techniques. We first address the differentiability of the bounds in section 5.1. With the differentiability, we are able to apply gradient-based optimization methods. These methods iteratively find a descent direction $p$ by ensuring that $\nabla f\left(x^{k}\right)^{T} p<0$, where $x^{k}$ is the solution of the $k$ th iteration and $f(x)$ is the function to be minimized. If $f$ is not continuously differentiable, $\nabla f\left(x^{k}\right)^{T} p<0$ does not imply that $p$ is a descent direction. Therefore, algorithms have trouble strictly decreasing the function value. We will check it and compute the derivative in section 5.1. However, none of the bounds is twice differentiable. This will also be discussed in section 5.1. Therefore, Newton's method cannot be used unless the Hessian (second derivative) is approximated by finite difference. Then quasi-Newton and nonlinear conjugate gradient methods are the remaining major candidates. Following Keerthi (2002), we examine quasi-Newton methods in section 5.2.

On the other hand, even if the second derivative is available, we still may not consider Newton's method. The advantage of using the secondorder information is to have fast convergence for a high precision. Keerthi (2002) pointed out that a high precision is not important on improving the test accuracy. Both Keerthi (2002) and experiments here indicate that under appropriate stopping conditions, the number of quasi-Newton iterations is already small. Furthermore, the computational cost on the Hessian matrix is expensive compared to the gradient. Thus, the effort of each Newton iteration is more than the quasi-Newton one. Details are set out in section 5.1.

5.1 Differentiability of Bounds for L1-SVM. We have proposed using equation 3.3 as the bound for L1-SVM. First, we denote it as $f\left(C, \sigma^{2}\right)$ and calculate its partial derivatives:

$$
\begin{aligned}
\frac{\partial f}{\partial C}= & \frac{\partial\left(R^{2}+\frac{\Delta}{C}\right)}{\partial C}\left(\|w\|^{2}+2 C \sum_{i=1}^{l} \xi_{i}\right) \\
& +\frac{\partial\left(\|w\|^{2}+2 C \sum_{i=1}^{l} \xi_{i}\right)}{\partial C}\left(R^{2}+\frac{\Delta}{C}\right), \\
\frac{\partial f}{\partial\left(\sigma^{2}\right)}= & \frac{\partial\left(R^{2}+\frac{\Delta}{C}\right)}{\partial\left(\sigma^{2}\right)}\left(\|w\|^{2}+2 C \sum_{i=1}^{l} \xi_{i}\right) \\
& +\frac{\partial\left(\|w\|^{2}+2 C \sum_{i=1}^{l} \xi_{i}\right)}{\partial\left(\sigma^{2}\right)}\left(R^{2}+\frac{\Delta}{C}\right) .
\end{aligned}
$$


For experiments in this section, we consider only $\Delta=1$. Note that $f\left(C, \sigma^{2}\right)$ may not be convex so we are solving a nonconvex optimization problem. We present an example in appendix $\mathrm{F}$.

The differentiability of both $R^{2}$ and $\|w\|^{2}+2 C \sum_{i=1}^{l} \xi_{i}$ relies on results of perturbation analysis of optimization problems. Theorem 4.1 of Bonnans and Shapiro (1998) gives a general result:

Theorem 3. For an optimization problem of the form $f^{*}(v)=\min _{\alpha \in A} f(\alpha, v)$ with optimization variable vector $\alpha$, parameter vector $v$, and $A$ independent of $v$, if for each $v$ there exists a unique $\alpha^{*}(v) \in A$ such that $f^{*}(v)=f\left(\alpha^{*}(v), v\right)$, then $\frac{d f^{*}}{d v}(v)$ exists and $\frac{\partial f^{*}}{\partial v_{i}}$ is given by $\frac{\partial f}{\partial v_{i}}\left(\alpha^{*}, v\right)$ for any parameter $v_{i}$.

Note that $\frac{d f^{*}}{d v}(v)$ does not depend on the existence of $\frac{d \alpha^{*}}{d v}(v)$. However, although $\frac{1}{2}\|w\|^{2}+C \sum_{i=1}^{l} \xi_{i}$ is the objective function of the primal L1-SVM, the theorem does not apply because the optimal $\xi$ may not be unique since the optimal $b$ is not unique when there is no free support vector, and constraints involve the kernel parameter $\sigma$, that is, $A$ is not independent of $v$. Therefore, we look at the dual problem as at any optimal solution,

$$
\|w\|^{2}+2 C \sum_{i=1}^{l} \xi_{i}=2\left(e^{T} \alpha-\frac{1}{2} \alpha^{T} Q \alpha\right)
$$

For the RBF kernel, if no training data are at the same point (i.e., $x_{i} \neq x_{j}$ ), $Q$ is positive definite (Micchelli, 1986). Then equation 1.6 is a strictly convex quadratic programming problem, so the optimal $\alpha$ is unique under any given parameters. Without much loss of generality from now on, we assume $x_{i} \neq x_{j}$. Then a remaining difficulty is that constraints of the dual form of L1-SVM are related to $C$. Therefore, we transform the dual to a problem whose constraints are independent of parameters.

From equation 1.6 , let $\alpha=C \bar{\alpha}$ :

$$
\begin{array}{cl}
\min _{\bar{\alpha}} & \frac{1}{2} \bar{\alpha}^{T} Q \bar{\alpha}-\frac{e^{T} \bar{\alpha}}{C} \\
\text { subject to } & 0 \leq \bar{\alpha} \leq 1, i=1, \ldots, l, \\
& y^{T} \bar{\alpha}=0 .
\end{array}
$$

Then,

$$
\frac{1}{2} \alpha^{T} Q \alpha-e^{T} \alpha=C^{2}\left(\frac{1}{2} \bar{\alpha} Q \bar{\alpha}-\frac{e^{T} \bar{\alpha}}{C}\right) .
$$


Finally, we can apply theorem 3 to equation 5.2 so

$$
\begin{aligned}
\frac{\partial\left(\frac{1}{2} \alpha^{T} Q \alpha-e^{T} \alpha\right)}{\partial C} & =2 C\left(\frac{1}{2} \bar{\alpha} Q \bar{\alpha}-\frac{e^{T} \bar{\alpha}}{C}\right)+C^{2}\left(\frac{e^{T} \bar{\alpha}}{C^{2}}\right) \\
& =\frac{2}{C}\left(\frac{1}{2} \alpha^{T} Q \alpha-e^{T} \alpha\right)+\frac{1}{C}\left(e^{T} \alpha\right) \\
& =\frac{1}{C}\left(\alpha^{T} Q \alpha-e^{T} \alpha\right) .
\end{aligned}
$$

With equation 5.1,

$$
\frac{\partial\left(\|w\|^{2}+2 C \sum_{i} \xi_{i}\right)}{\partial C}=\frac{2}{C}\left(e^{T} \alpha-\alpha^{T} Q \alpha\right)=2 \sum \xi_{i} .
$$

Similarly,

$$
\begin{aligned}
& \frac{\partial\left(\|w\|^{2}+2 C \sum \xi_{i}\right)}{\partial\left(\sigma^{2}\right)}=-\sum \alpha_{i} \alpha_{j} y_{i} y_{j} \frac{\partial K\left(x_{i}, x_{j}\right)}{\partial\left(\sigma^{2}\right)}, \\
& \frac{\partial R^{2}+\Delta / C}{\partial C}=\frac{-\Delta}{C^{2}}, \frac{\partial R^{2}+\Delta / C}{\partial\left(\sigma^{2}\right)}=-\sum \beta_{i} \beta_{j} \frac{\partial K\left(x_{i}, x_{j}\right)}{\partial\left(\sigma^{2}\right)},
\end{aligned}
$$

where

$$
\frac{\partial K\left(x_{i}, x_{j}\right)}{\partial\left(\sigma^{2}\right)}=K\left(x_{i}, x_{j}\right) \frac{\left\|x_{i}-x_{j}\right\|^{2}}{2 \sigma^{4}} .
$$

Note that the differentiability is special to the RBF kernel since the uniqueness of $\alpha$ may not hold in the hyperspace for other kernels. However, for L2-SVM, as the Hessian of equations 1.8 and 1.11 is positive definite, they always have unique solutions. Thus, RM bound for L2-SVM is differentiable no matter which kernel is used.

In contrast to equation 3.3, the derivatives of other bounds may require the partial derivatives of $\alpha$ with respect to the hyperparameters $C$ and $\sigma^{2}$. This dependency raises two drawbacks: nondifferentiability and high computational cost. For the nondifferentiability, we give two examples for L1SVM and L2-SVM in appendixes F and G, respectively, showing that $\frac{\partial \alpha}{\partial C}$ may not be differentiable. To compute the derivatives of equation 3.3, we need to compute equations 5.4 through 5.6. Since only nonzero $\alpha_{i}$ contribute to equation 5.5, the computational cost is $O\left(l_{s v}^{2} n\right)$, where $l_{s v}$ denotes the number of nonzero elements in $\alpha$. The situation of equation 5.6 is similar. However, as shown in Chapelle et al. (2002), under the assumption that the support vectors do not change, to compute $\frac{\partial \alpha}{\partial v}$ where $v$ denotes hyperparameters requires the inverse of $Q_{s v}$, which is the submatrix of $Q$ involving with support vectors, and the inversion of $Q_{s v}$ usually takes $O\left(l_{s v}^{3}\right)$ time. 
Although we have differentiable bounds for both L1- and L2-SVMs, they are not twice differentiable since the second-order derivatives require $\frac{\partial \alpha}{\partial v}$ also. For example, the second-order derivative of our RM bound contains the partial derivative of equation 5.4 with respect to $C$, which is

$$
\frac{\partial}{\partial C} \frac{2}{C}\left(e^{T} \alpha-\alpha^{T} Q \alpha\right)=-\frac{2}{C^{2}}\left(e^{T} \alpha-\alpha^{T} Q \alpha\right)+\frac{2}{C}\left(e^{T}-2 \alpha^{T} Q\right) \frac{\partial \alpha}{\partial C},
$$

dependent with the nondifferentiable term $\frac{\partial \alpha}{\partial C}$. Similarly, the need of $\frac{\partial \alpha}{\partial C}$ also forbids us using second derivatives due to its expensive computational cost discussed before.

5.2 Quasi-Newton Methods. Following earlier experiments, we search on the $\left(\ln C, \ln \sigma^{2}\right)$ space. An advantage is that the optimization problem becomes unconstrained. Otherwise, $C \geq 0$ and the property $\sigma^{2}=(-\sigma)^{2}$ cause problems. However, practically, we still have to specify upper and lower bounds for $\ln C$ and $\ln \sigma^{2}$. As in section 4, we restrict them to be in $[-10,10]$. Once the optimization procedure has reached the boundary and still tends to move out, we stop it.

Working on the $\left(\ln C, \ln \sigma^{2}\right)$ space, the gradient is also different. We must modify equations 5.4 through 5.6 according to the following chain rules:

$$
\frac{\partial}{\partial \ln C}=\frac{\partial}{\partial C} \times C, \text { and } \frac{\partial}{\partial \ln \sigma^{2}}=\frac{\partial}{\partial\left(\sigma^{2}\right)} \times \sigma^{2} .
$$

We consider the Broyden-Fletcher-Goldfarb-Shanno (BFGS) quasiNewton method (see Nocedal \& Wright, 1999) which has the following procedure: Assume $x^{k}$ is the current iterate and $f(x)$ is the function to be minimized:

1. Compute a search direction $p=-H_{k} \nabla f\left(x^{k}\right)$.

2. Find $x^{k+1}=x^{k}+\lambda p$ using a line search to ensure sufficient decrease.

3. Obtain $H_{k+1}$ by

$$
H_{k+1}=\left(I-\frac{s y^{T}}{y^{T} S}\right) H_{k}\left(I-\frac{y s^{T}}{y^{T} S}\right)+\frac{s s^{T}}{y^{T} S},
$$

where

$$
s=x^{k+1}-x^{k} \text { and } y=\nabla f\left(x^{k+1}\right)-\nabla f\left(x^{k}\right) .
$$


Here, $H_{k}$ serves as the inverse of an approximate Hessian. The sufficient decrease by the line search usually means

$$
f\left(x^{k}+\lambda p\right) \leq f\left(x^{k}\right)+\sigma_{1} \lambda \nabla f\left(x^{k}\right)^{T} p
$$

where $0<\sigma_{1}<1$ is a positive constant. Since $p=-H_{k} \nabla f\left(x^{k}\right)$, we need $H_{k}$ to be positive definite to ensure that $p$ is a descent direction. A good property of the BFGS formula is that $H_{k+1}$ inherits the positive definiteness of $H_{k}$ as long as $y^{T} s>0$. The condition $y^{T} s>0$ is guaranteed to hold if the initial Hessian is positive definite (e.g., the identity) and the step size is determined by satisfying the second Wolfe condition:

$$
\nabla f\left(x^{k}+\lambda p\right) \geq \sigma_{2} \nabla f\left(x^{k}\right)^{T} p
$$

where $0<\sigma_{1}<\sigma_{2}<1$. Note that equation 5.7 is usually called the first Wolfe condition.

The main disadvantage of considering equation 5.8 is that the line search becomes more complicated. In addition, equation 5.8 involves the calculation of $\nabla f\left(x^{k}+\lambda p\right)$ so for each trial step size, a gradient evaluation is needed. Though $\nabla f\left(x^{k}+\lambda p\right)$ is easily computed once $f\left(x^{k}+\lambda p\right)$ is computed (as pointed out in Keerthi, 2002), this still contributes some additional cost.

We consider an alternative approach to avoid the more complicated line search. If $y^{T} s<0, H_{k}$ is not updated. More specifically, $H_{k+1}$ is determined by

$$
H_{k+1}= \begin{cases}\left(I-\frac{s y^{T}}{y^{T} s}\right) H_{k}\left(I-\frac{y s^{T}}{y^{T} S}\right)+\frac{s S^{T}}{y^{T} S} & \text { if } y^{T} s>\eta \\ H_{k} & \text { otherwise }\end{cases}
$$

where $\eta$ is usually a small constant. Here, we simply use $\eta=0$. Then the second Wolfe condition is not needed. The disadvantage of equation 5.9 is that it may lengthen the procedure if in many iterations, $H_{k}$ is not updated. Here, we do not have this problem as in our experiments, for only very few iterations $H_{k}$ is not updated.

It is unfortunate that the global convergence of BFGS algorithm for nonconvex problems is still an open issue. Existing convergence results (Li \& Fukushima, 2001) require either further modification of the algorithm or more conditions on the function.

Regarding different trials of step size to ensure the sufficient decrease condition equation 5.7, we can simply find the largest value in a set $\left\{\gamma^{i} \mid\right.$ $i=0,1, \ldots\}$ such that equation 5.7 holds $(\gamma=1 / 2$ used in this letter). More advanced techniques such as quadratic or cubic interpolation (Nocedal \& Wright, 1999) can also be used. However, we do not recommend them here, as for most iterations, an initial step size $\lambda=1$ already satisfies equation 5.8. Also note that in early iterations, the search direction $p$ may be a vector that 
is large in size, so using the initial $\lambda=1$, sometimes $x^{k}+p$ is far beyond the region considered. Thus, numerical instability may occur. Therefore, if $x^{k}+\lambda p$ is outside the $[-10,10] \times[-10,10]$ region, we project it back by

$$
P\left(x_{i}^{k}+\lambda p_{i}\right)=\max \left(-10, \min \left(x_{i}^{k}+\lambda p_{i}, 10\right)\right) .
$$

We further avoid a step size that is too large by requiring the initial $\lambda$ to satisfy

$$
\left\|P\left(x^{k}+\lambda p\right)-x^{k}\right\| \leq 2 .
$$

This reduces the chance of going to a wrong region in the beginning.

5.3 Initial Points and Stopping Criteria. Keerthi (2002) proposed using either

$$
\ln C=0, \ln \sigma^{2}=0
$$

or

$$
\ln C=0, \ln \sigma^{2}=\ln \hat{R}^{2}
$$

as the initial points, where $\hat{R}^{2}$ is the radius of the smallest sphere that contains all training examples in their original space. Keerthi (2002) notes that the latter performs better. However, from experiments, we observed that both settings are appropriate. Thus, in Figures 2 through 4, we use equation 5.11 as the initial point in our implementation. Indeed most points on the $\left(\ln C, \ln \sigma^{2}\right)$ plane as initials lead to the convergence toward the local minimum, which we would like to identify. The only exception is that when initial $\ln C$ and $\ln \sigma^{2}$ are too large or too small, the procedure goes toward other local minimum. Another reason to avoid larger initial $\ln C$ and $\ln \sigma^{2}$ is that methods used to solve each individual SVM usually take more time on such parameters.

For the stopping condition, Keerthi (2002) considers

$$
\left|f\left(x^{k+1}\right)-f\left(x^{k}\right)\right| \leq 10^{-5} f\left(x^{k}\right) .
$$

However, the value of $f\left(x^{k}\right)$ ranges too much to be applicable to our procedure. A large $f\left(x^{k}\right)$ causes an early stop of our procedure, whereas a small $f\left(x^{k}\right)$ easily leads to too many iterations. Instead, we check the gradient, as frequently used in many other iterative procedures. Following Lin and Moré (1999), we use

$$
\frac{\left\|\nabla f\left(x^{k}\right)\right\|}{\left\|\nabla f\left(x^{0}\right)\right\|} \leq 10^{-3} \text { or }\left\|\nabla f\left(x^{k}\right)\right\| \leq 10^{-3},
$$

where $x^{0}$ is the initial solution. Usually the relative condition is achieved first. 
5.4 Implementation. We use LIBSVM (Chang \& Lin, 2001b), which implements a decomposition method, to calculate $\|w\|^{2},\|\tilde{w}\|^{2}, R^{2}$, and $\tilde{R}^{2}$. The quasi-Newton implementation is written in Python by modifying the optimization subroutine by Travis Oliphant (available in Scientific Python online at http://www.scipy.org). Tools such as numerical Python are employed so it is easy to conduct matrix and vector operations. The Python program is then linked to LIBSVM through its Python interface. Some advantages of using Python include the powerful data preprocessing and the easy link with other graphic tools. The computational experiments for this section were done on a Pentium III-1000 with 1024 MB RAM using the gcc compiler.

We keep all the default settings of LIBSVM except using a smaller stopping tolerance $10^{-6}$ (default $10^{-3}$ ) and increasing the cache size. Keerthi (2002) pointed out that near the minimizer, $\|\nabla f\|$ is small so the error associated with finding $\|w\|^{2},\|\tilde{w}\|^{2}, R^{2}$, or $\tilde{R}^{2}$ may strongly affect the search direction. We have the same observation so use a smaller stopping tolerance.

For our implementation, solving each of $\|w\|^{2},\|\tilde{w}\|^{2}, R^{2}$, or $\tilde{R}^{2}$ is considered an independent problem. In the future, $\|w\|^{2}$ and $R^{2}\left(\|\tilde{w}\|^{2}\right.$ and $\left.\tilde{R}^{2}\right)$ should be considered together. How to effectively pass information under one parameter set to another is also worthy of investigation.

5.5 Experiments. We compare the quasi-Newton implementation for L1- and L2-SVM. For L1-SVM, we use equation 3.3 with $\Delta=1$. The same five problems are considered. To demonstrate the viability for solving large problems, we include the problem ijcnn1, which has 49,990 training and 91,701 testing samples in two classes. It is from the first problem of IJCNN challenge 2001 (Prokhorov, 2001). Note that we use the winner's transformation of raw data (Chang \& Lin, 2001a).

Tables 7 and 8 present the results using different initial points: $(0,0)$ and $(-3,-3)$. We list the number of function and gradient evaluations, the optimal $\left(\ln C, \ln \sigma^{2}\right)$, and the test accuracy. Results for L2-SVM are consistent with Table 1 of Keerthi (2002). Note that the number of gradient evaluations is the same as the number of quasi-Newton iterations. Hence, it can be seen that the average number of line searches in each iteration is very close to one. For problems image, splice, and tree, using L1-SVM, the algorithm reaches a point with $\ln C=10$. At that point, there are no bounded support vectors, so we can set the resulting $\ln C$ to the largest element of the dual variable $\alpha$ without affecting the model produced. Actually in the final iterations for these problems, there are already no bounded support vectors in the models. But unlike the grid search in section 4 , where we can stop increasing $C$ for the same $\sigma^{2}$, here $C$ and $\sigma^{2}$ can both change in a single iteration and must be considered together. We have not been able to develop early stopping criteria for such a situation so decided to let the algorithm continue until it reaches the boundary. Thus, for image and waveform, L1-SVM takes more iterations than L2-SVM. For splice, L2-SVM also reaches $\ln C=10$. Overall, 
Table 7: Results of the Experiments Using RM Bounds with $\left(\ln C, \ln \sigma^{2}\right)=(0,0)$ as the Initial Solution.

\begin{tabular}{|c|c|c|c|c|}
\hline & \multicolumn{4}{|c|}{ L1 } \\
\hline & \#fun & \#grad & $\left(\ln C, \ln \sigma^{2}\right)$ & Accuracy \\
\hline banana & 9 & 6 & $(-0.83,-1.69)$ & 88.96 \\
\hline image & 17 & 13 & $(2.99,-1.20)$ & 96.24 \\
\hline splice & 13 & 12 & $(1.09,3.07)$ & 89.84 \\
\hline tree & 8 & 8 & $(-1.71,0.53)$ & 86.50 \\
\hline waveform & 16 & 13 & $(1.20,1.38)$ & 88.57 \\
\hline \multirow[t]{3}{*}{ ijcnn1 } & 9 & 9 & $(2.06,-4.05)$ & 97.09 \\
\hline & \multicolumn{4}{|c|}{ L2 } \\
\hline & \#fun & \#grad & $\left(\ln C, \ln \sigma^{2}\right)$ & Accuracy \\
\hline banana & 8 & 5 & $(-0.91,-1.64)$ & 88.53 \\
\hline image & 11 & 6 & $(0.44,-0.29)$ & 97.03 \\
\hline splice & 21 & 19 & $(10.00,3.07)$ & 89.84 \\
\hline tree & 8 & 8 & $(-1.86,0.97)$ & 86.54 \\
\hline waveform & 8 & 7 & $(-0.50,2.36)$ & 89.83 \\
\hline ijcnn1 & 7 & 7 & $(-0.10,-2.58)$ & 97.83 \\
\hline
\end{tabular}

Note: The bound used for L1-SVM is equation 3.3 with $\Delta=1$. \#fun and \#grad represent the number of function and gradient evaluations, respectively.

Table 8: Results of the Experiments Using RM Bounds with $\left(\ln C, \ln \sigma^{2}\right)=$ $(-3,-3)$ as the Initial Solution.

\begin{tabular}{|c|c|c|c|c|}
\hline & \multicolumn{4}{|c|}{ L1 } \\
\hline & \#fun & \#grad & $\left(\ln C, \ln \sigma^{2}\right)$ & Accuracy \\
\hline banana & 8 & 7 & $(-0.83,-1.69)$ & 88.96 \\
\hline image & 25 & 23 & $(2.99,-1.20)$ & 96.24 \\
\hline splice & 10 & 10 & $(-10.00,-3.00)$ & 52.00 \\
\hline tree & 13 & 11 & $(-1.71,0.53)$ & 86.50 \\
\hline waveform & 10 & 10 & $(-10.00,-3.00)$ & 67.07 \\
\hline \multirow[t]{3}{*}{ ijcnn1 } & 13 & 12 & $(2.06,-4.05)$ & 97.09 \\
\hline & \multicolumn{4}{|c|}{ L2 } \\
\hline & \#fun & \#grad & $\left(\ln C, \ln \sigma^{2}\right)$ & Accuracy \\
\hline banana & 8 & 7 & $(-0.91,-1.64)$ & 88.53 \\
\hline image & 8 & 8 & $(0.44,-0.29)$ & 97.03 \\
\hline splice & 14 & 14 & $(10.00,-2.68)$ & 56.92 \\
\hline tree & 7 & 7 & $(-1.86,0.97)$ & 86.54 \\
\hline waveform & 1 & 1 & $(-3.00,-3.00)$ & 67.07 \\
\hline ijcnn1 & 6 & 6 & $(-0.10,-2.58)$ & 97.83 \\
\hline
\end{tabular}

Note: The bound used for L1-SVM is equation 3.3 with $\Delta=1$. \#fun and \#grad represent the number of function and gradient evaluations, respectively. 
except this difference, in terms of accuracy as well as computational cost, the bound for L1-SVM is competitive with that for L2-SVM.

For the large problem ijcnn1, RM bounds for both L1- and L2-SVM reach points with error rate around 0.0291 and 0.0217 , respectively. Unfortunately, this is worse than 0.0141 by $C=16$ and $\sigma^{2}=0.125$ using cross validation (Chang \& Lin, 2001a). In addition, the number of support vectors is very different. There are about 17,000 support vectors compared to 3370 in Chang and Lin (2001a). However, the total computational time is around 26,000 seconds (using the default $10^{-3}$ as the stopping tolerance of LIBSVM), much shorter than doing cross validation.

When $(-3,-3)$ is used as the initial solution in Table 8 for splice and waveform, the algorithm does not converge to desired points. This seems to show that for these problems, $(-3,-3)$ is already too far away from the good region. For L1 bound 3.3, the vertical contour lines in the bottom of Figures 2 through 5 show that the bound is almost independent of $\sigma$ when $\sigma^{2} \rightarrow 0$. In appendix $\mathrm{D}$, we show that when $\sigma^{2} \rightarrow 0$, the algorithm tends to find a too large or too small $C$. Thus, we should avoid a too small $\sigma^{2}$ as the initial solution.

To further compare bounds for L1- and L2-SVM, in Figures 2 through 5, we present contour plots of tree, waveform, and splice, with searching paths on them. Note that in the L1 case of Figure 3, the final solution is projected from $(8.87,1.38)$ to $(1.25,1.38)$ because there are no bounded support vectors. Figure 5 is an example that the algorithm does not converge to the desired region, where the initial solution is too far away from the good region.

\section{Discussion and Conclusion}

6.1 SVM Model Selection Software. People frequently say that an advantage of SVM over neural networks is that SVM solves a convex problem. However, this is true only in the sense that parameters are given. Once model selection is considered, we still deal with difficult nonconvex problems.
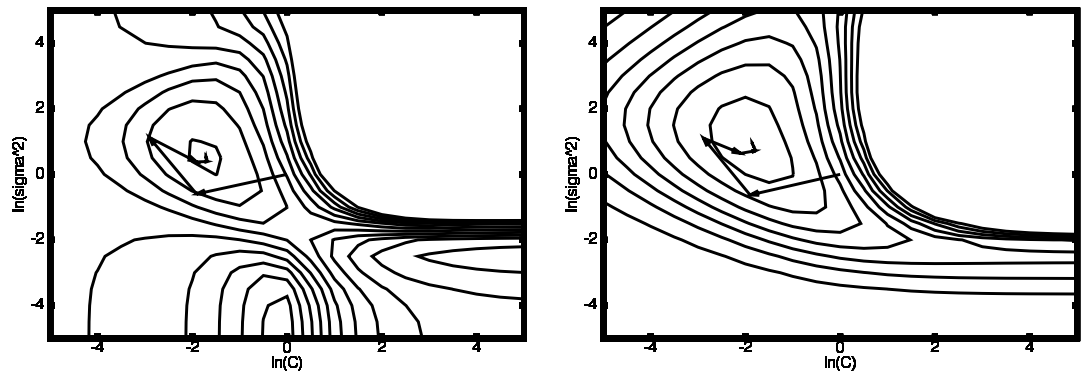

Figure 2: Contour plots and searching paths of tree. (Left) L1 bound. (Right) L2 bound. The initial solution is $(0,0)$. 

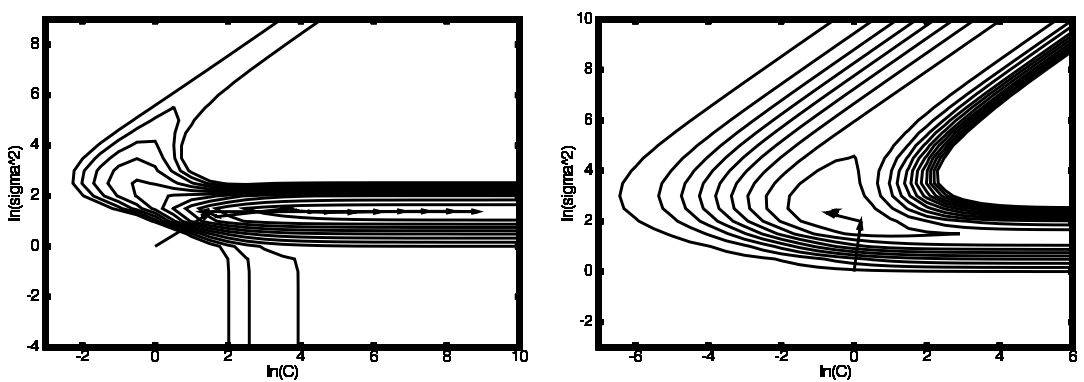

Figure 3: Contour plots and searching paths of waveform. (Left) L1 bound. (Right) L2 bound. The initial solution is $(0,0)$. The final solution for the L1 bound is projected from $(8.87,1.38)$ to $(1.25,1.38)$.
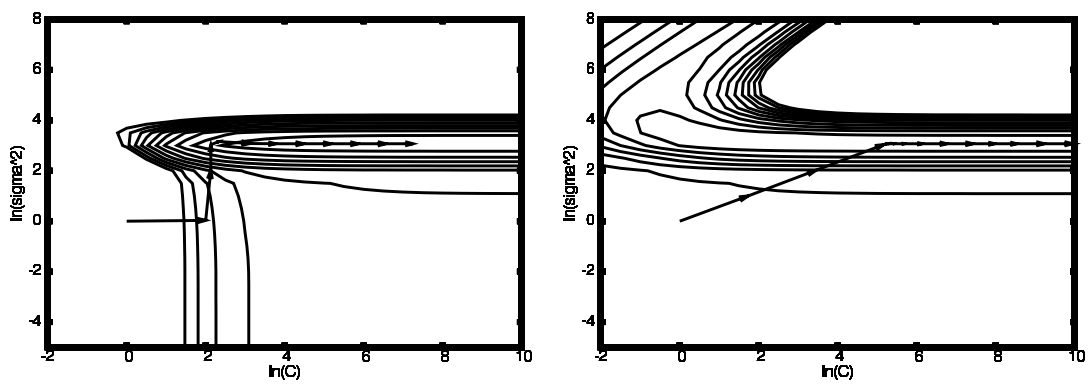

Figure 4: Contour plots and searching paths of splice. (Left) L1 bound. (Right) L2 bound. The initial solution is $(0,0)$.
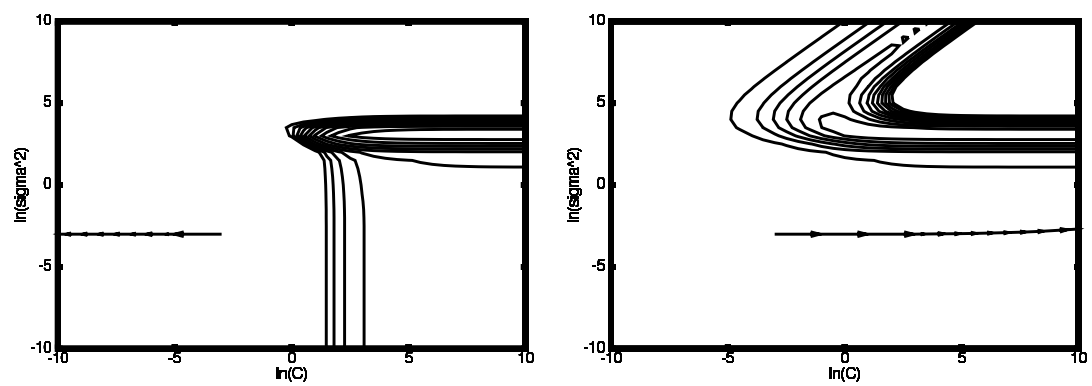

Figure 5: Contour plots and searching paths of splice. (Left) L1 bound. (Right) L2 bound. The initial solution is $(-3,-3)$. Quasi-Newton iterations fail to converge to the desired regions. 
Therefore, it is fair to say that SVM elegantly separates things to different stages where the innermost one solves simple convex problems. Easy-to-use implementations for solving these convex problems have been available. However, if we integrate everything together, the SVM software will be more complicated. In particular, there may be a need to provide different model selection strategies. For small problems where cross validation is affordable, a complete search may be more reliable. But for large problems, using heuristic bounds is essential. Whether users can adapt to these different techniques and conduct appropriate model selection remains a main challenge for SVM software development.

6.2 Remaining Implementation Issues. As the search direction of quasiNewton method depends on accurately solving each SVM dual, we have used a smaller stopping tolerance for LIBSVM. However, this also lengthen the total computational time. The requirement of high accuracy on solving SVM duals happens in final iterations of the quasi-Newton procedure. How to adapt the stopping tolerance for SVM duals in the quasi-Newton procedure effectively is a future issue.

6.3 Heuristic Bounds for Other Problems. In this article, we discuss only two-class classification SVM. However, it is possible to extend our results and apply similar ideas on multiclass classification problems and SVM regression (Chang \& Lin, 2003). Moreover, the heuristic bounds for L1-SVM developed in this article may be useful for tuning many hyperparameters, such as doing automatic relevance determination of input features.

\section{Appendix A: Proof of Theorem 1}

We introduce some required preliminaries before the proof. In fact, equations 1.3 and 1.11 are a dual form of the following problem:

$$
\begin{gathered}
\min _{a, R} R^{2} \\
\text { subject to } R^{2}-\left\|\phi\left(x_{i}\right)-a\right\|^{2} \geq 0, i=1, \ldots, l,
\end{gathered}
$$

where the vector $a$ is the center of the sphere. From the KKT condition, we have

$$
a=\sum_{j=1}^{l} \beta_{j} \phi\left(x_{j}\right),
$$

and if $\beta_{i} \neq 0$,

$$
R^{2}-\left\|\phi\left(x_{i}\right)-a\right\|^{2}=0 .
$$

We then need a technical lemma: 
Lemma 2. For any kernel, the vector $\beta$ in dual form of $12 p t R^{2}$ satisfies $\beta^{T} \beta \leq$ $1 / 2$.

Proof. It suffices to show that $\beta_{i} \leq 1 / 2$ for $i=1, \ldots, l$. If it is true, with $\sum_{i=1}^{l} \beta_{i}=1$ and $\beta_{i} \geq 0$ for $i=1, \ldots, l$, we have $\beta^{T} \beta \leq 1 / 2$.

Assume that $\beta_{i} \neq 0$ for some $i$. Equations A.1 and A.2 imply

$$
\begin{aligned}
R^{2} & =\left\|\phi\left(x_{i}\right)-a\right\|^{2} \\
& =\left\|\phi\left(x_{i}\right)-\sum_{j=1}^{l} \beta_{j} \phi\left(x_{j}\right)\right\|^{2} \\
& =\left(1-\beta_{i}\right)^{2}\left\|\phi\left(x_{i}\right)-\sum_{j \neq i} \frac{\beta_{j}}{1-\beta_{i}} \phi\left(x_{j}\right)\right\|^{2} \\
& \equiv\left(1-\beta_{i}\right)^{2}\left\|\phi\left(x_{i}\right)-z\right\|^{2} .
\end{aligned}
$$

Since $\beta_{j} \geq 0$ and $\sum_{j \neq i} \frac{\beta_{j}}{1-\beta_{i}}=1, z$ is in the convex hull of $\phi\left(x_{j}\right), j \neq i$. Thus,

$$
R^{2} \leq\left(1-\beta_{i}\right)^{2} D^{2}=4\left(1-\beta_{i}\right)^{2} R^{2}
$$

so $\beta_{i} \leq 1 / 2$.

We now return to the proof of theorem 1. First, we have

$$
\beta^{T}\left(K+\frac{I}{C}\right) \beta \geq \tilde{\beta}^{T}\left(K+\frac{I}{C}\right) \tilde{\beta} \geq \beta^{T} K \beta+\frac{\tilde{\beta} \tilde{\beta}}{C},
$$

where $\beta$ and $\tilde{\beta}$ are optimal solutions of equations 1.3 and 1.11, respectively. Because $K_{i i}=1$ and $K_{i j} \geq 0$,

$$
\tilde{\beta}^{T} K \tilde{\beta} \geq \beta^{T} K \beta \geq \beta^{T} \beta \geq \tilde{\beta}^{T} \tilde{\beta} .
$$

By the differentiability discussed in section 5.1, we consider the partial derivative of $\tilde{R}^{2} / R^{2}+\frac{0.5}{C}$ :

$$
\frac{\partial}{\partial C}\left(\frac{\tilde{R}^{2}}{R^{2}+\frac{0.5}{C}}\right)=\left(\left(R^{2}+\frac{0.5}{C}\right) \frac{\partial \tilde{R}^{2}}{\partial C}-\left(\frac{-0.5}{C^{2}}\right) \tilde{R}^{2}\right) /\left(R^{2}+\frac{0.5}{C}\right)^{2} .
$$

We have

$$
\begin{aligned}
& \left(R^{2}+\frac{0.5}{C}\right) \frac{\partial \tilde{R}^{2}}{\partial C}-\left(\frac{-0.5}{C^{2}}\right) \tilde{R}^{2} \\
& =R^{2} \frac{\partial \tilde{R}^{2}}{\partial C}+\frac{0.5}{C^{2}}\left(C \frac{\partial \tilde{R}^{2}}{\partial C}+\tilde{R}^{2}\right)
\end{aligned}
$$




$$
\begin{aligned}
& =R^{2} \frac{\partial \tilde{R}^{2}}{\partial C}+\frac{0.5}{C^{2}}\left(\frac{-1+\tilde{\beta}^{T} \tilde{\beta}}{C}+1+\frac{1}{C}-\tilde{\beta}^{T}\left(K+\frac{I}{C}\right) \tilde{\beta}\right) \\
& =\left(1-\beta^{T} K \beta\right)\left(\frac{-1}{C^{2}}\right)\left(1-\tilde{\beta}^{T} \tilde{\beta}\right)+\frac{0.5}{C^{2}}\left(1-\tilde{\beta}^{T} K \tilde{\beta}\right) \\
& =\frac{1}{C^{2}}\left(\frac{-1}{2}+\beta^{T} K \beta+\tilde{\beta}^{T} \tilde{\beta}-\left(\beta^{T} K \beta\right)\left(\tilde{\beta}^{T} \tilde{\beta}\right)-0.5 \tilde{\beta}^{T} K \tilde{\beta}\right) \\
& \leq \frac{1}{C^{2}}\left(\frac{-1}{2}+0.5 \beta^{T} K \beta+\left(1-\beta^{T} K \beta\right) \tilde{\beta}^{T} \tilde{\beta}\right) \\
& =\frac{1}{C^{2}}\left(\left(\frac{-1}{2}+\tilde{\beta}^{T} \tilde{\beta}\right)\left(1-\beta^{T} K \beta\right)\right) \\
& \leq 0,
\end{aligned}
$$

since $\tilde{\beta}^{T} \tilde{\beta} \leq 1 / 2$ from lemma 2 . Therefore, with equation 2.6 , we conclude that $\tilde{R}^{2} /\left(R^{2}+\frac{0.5}{C}\right)$ monotonically decreases to 1 as $C \rightarrow \infty$ and

$$
\left(R^{2}+\frac{0.5}{C}\right)\|\tilde{w}\|^{2} \leq \tilde{R}^{2}\|\tilde{w}\|^{2}, \forall C .
$$

\section{Appendix B: Proof of Lemma 1}

Define

$$
\begin{aligned}
\Lambda_{p} & \equiv\left\{\lambda=\left(\lambda_{1}, \lambda_{2}, \ldots, \lambda_{l}\right) \mid \lambda_{p}=-1, \sum_{i=1}^{l} \lambda_{i}=0, \alpha_{i}^{0}+y_{i} y_{p} \alpha_{p}^{0} \lambda_{i} \geq 0\right\}, \\
\Lambda_{p}^{+} & \equiv\left\{\lambda \in \Lambda_{p} \mid \lambda_{i} \geq 0, \forall i \neq p\right\} .
\end{aligned}
$$

For any $\lambda \in \Lambda_{p}^{+}$,

$$
\left(\sum_{i=1}^{l} \lambda_{i} \phi\left(x_{i}\right)\right)^{2}=\left\|\phi(x)_{p}-\sum_{i \notin p} \lambda_{i} \phi\left(x_{i}\right)\right\|^{2} \leq D^{2},
$$

where $D$ is the diameter of the smallest ball containing all training data.

Select $\lambda^{*} \in \Lambda_{p}^{+}$by

$$
\lambda_{i}^{*}= \begin{cases}-1 & \text { if } i=p, \\ \frac{1}{l_{s v 1}-1} & \text { if } y_{i}=y_{p}=1, \\ \frac{1}{l_{s v 2}-1} & \text { if } y_{i}=y_{p}=-1, \\ 0 & \text { if } y_{i} \neq y_{p} .\end{cases}
$$


Now

$$
\tilde{S}_{p}^{2} \equiv \min \left\{\left(\sum_{i=1}^{l} \lambda_{i} \tilde{z}_{i}\right)^{2} \mid \lambda_{p}=-1, \sum_{i=1}^{l} \lambda_{i}=0, \alpha_{i}^{0}+y_{i} y_{p} \alpha_{p}^{0} \lambda_{i} \geq 0\right\},
$$

where $\tilde{z}_{i}$ is defined in equation 2.2 .

Since $\Lambda_{p}^{+}$is a subset of $\Lambda_{p}, \lambda^{*} \in \Lambda_{p}$. With equation B.1, we obtain

$$
\begin{aligned}
\tilde{S}_{p}^{2} & \leq\left(\sum_{i=1}^{l} \lambda_{i}^{*} \tilde{z}_{i}\right)^{2} \\
& =\left(\sum_{i=1}^{l} \lambda_{i}^{*} \phi\left(x_{i}\right)\right)^{2}+\left(\sum_{i=1}^{l} \frac{\lambda_{i}^{*} e_{i}}{\sqrt{C}}\right)^{2} \\
& \leq D^{2}+\frac{1}{C}+ \begin{cases}\frac{1}{\left(l_{s v 1}-1\right) C} & \text { if } y_{p}=1 \\
\frac{1}{\left(l_{s v 2}-1\right) C} & \text { if } y_{p}=-1\end{cases}
\end{aligned}
$$

\section{Appendix C: Proof of Equations 2.15 Through 2.17}

Consider the following problem:

$$
\begin{array}{cl}
\max _{\alpha} & e^{T} \alpha-\frac{\alpha^{T} \alpha}{2 C} \\
\text { subject to } & 0 \leq \alpha_{i}, i=1, \ldots, l, \\
y^{T} \alpha=0 .
\end{array}
$$

We can easily see

$$
\bar{\alpha}_{i}= \begin{cases}\frac{2 l_{2}}{l} C & \text { if } y_{i}=1, \\ \frac{2 l_{1}}{l} C & \text { if } y_{i}=-1,\end{cases}
$$

is an optimal solution. It satisfies the Karush-Kuhn-Tucker (KKT) condition that there is a number

$$
b=\frac{l_{1}-l_{2}}{l} \text { such that } \frac{\alpha_{i}}{C}-1+b y_{i}=0, i=1, \ldots, l .
$$

Under any fixed $C$, we assume $\alpha(\sigma)$ is the optimal solution of the dual L2SVM, equation 1.8, and $Q(\sigma)_{i j}=e^{-\left\|x_{i}-x_{j}\right\|^{2} /\left(2 \sigma^{2}\right)}$. As equations C.1 and 1.8 have the same feasible set and $Q(\sigma)$ is positive semidefinite,

$$
\begin{aligned}
e^{T} \bar{\alpha}-\frac{\bar{\alpha}^{T} \bar{\alpha}}{2 C} & \geq e^{T} \alpha(\sigma)-\frac{1}{2} \alpha(\sigma)^{T}\left(Q(\sigma)+\frac{I}{C}\right) \alpha(\sigma) \\
& \geq e^{T} \bar{\alpha}-\frac{1}{2} \bar{\alpha}^{T}\left(Q+\frac{I}{C}\right) \bar{\alpha}
\end{aligned}
$$


When $\sigma \rightarrow \infty, Q(\sigma) \rightarrow y y^{T}$ so with $y^{T} \bar{\alpha}=0$,

$$
\lim _{\sigma \rightarrow \infty}\|\tilde{w}\|^{2}=2\left(e^{T} \bar{\alpha}-\frac{\bar{\alpha}^{T} \bar{\alpha}}{2 C}\right)=\frac{4 C l_{1} l_{2}}{l} .
$$

Similarly, we can show

$$
\lim _{\sigma \rightarrow \infty} \tilde{R}^{2}=\frac{1}{C}\left(1-\frac{1}{l}\right)
$$

Thus, if $l \gg 1$, when $\sigma \rightarrow \infty$,

$$
\tilde{R}^{2}\|\tilde{w}\|^{2} \approx \frac{4 l_{1} l_{2}}{l}
$$

We can also prove that when $\sigma \rightarrow \infty, R^{2} \rightarrow 0$. Then, equations 2.16 and 2.17 follow.

Appendix D: Limit Behavior of Equation 3.3 When $\sigma^{2} \rightarrow 0$

From Keerthi and Lin (forthcoming), the optimal solution of equation 1.6 when $\sigma^{2} \rightarrow 0$ is:

$$
\alpha^{-}=\left\{\begin{array}{ll}
C_{\lim } & \text { if } C \geq C_{\lim } \\
C & \text { if } C<C_{\lim }
\end{array}, \quad \alpha^{+}=\left\{\begin{array}{ll}
\frac{2 l_{2}}{l} & \text { if } C \geq C_{\lim } \\
\frac{l_{2} C}{l_{1}} & \text { if } C<C_{\lim }
\end{array},\right.\right.
$$

where $\alpha_{i}=\alpha^{+}$if $y_{i}=+1, \alpha_{i}=\alpha^{-}$if $y_{i}=-1, C_{\lim }=\frac{2 l_{1}}{l}$, and $l_{1}>l_{2}+1>2$. Here $l_{1}, l_{2}$ is number of training examples which $y_{i}=+1$ and $y_{i}=-1$ respectively.

It is easy to show that when $\sigma^{2} \rightarrow 0$, the optimal solution of equation 1.3 is $\beta=\frac{e}{l}$, where $e \in R^{l}$ is a vector with all its elements 1 . Thus, when $\sigma^{2} \rightarrow 0$, equation 3.3 becomes

$$
\begin{aligned}
& \left(\frac{l-1}{l}+\frac{\Delta}{C}\right)\left(\frac{4 l_{1} l_{2}}{l}\right) \quad \text { if } C \geq C_{\lim }, \\
& \left(\frac{l-1}{l}+\frac{\Delta}{C}\right)\left(4 l_{2} C-l_{2} C^{2}-\frac{l_{2}^{2} C^{2}}{l_{1}}\right) \quad \text { if } C<C_{\lim } .
\end{aligned}
$$

Thus, when $C<C_{\text {lim }}$, equation 3.3 is a concave function on $C$; when $C \geq C_{\text {lim }}$, equation 3.3 is a decreasing function on $C$. This explains the phenomenon that when $\sigma^{2} \rightarrow 0$, the algorithm will find a too large or too small $C$ in section 5 . 


\section{Appendix E: Proof of Theorem 2}

If $x$ and $x^{\prime}$ are global optima of $f_{1}(x)$ and $f_{2}(x)$, respectively, using $g>0$,

$$
\begin{aligned}
& f_{2}\left(x^{\prime}\right) g\left(x^{\prime}\right) \leq f_{2}(x) g(x), \text { and } \\
& f_{1}(x) g(x) \leq f_{1}\left(x^{\prime}\right) g\left(x^{\prime}\right) .
\end{aligned}
$$

Therefore,

$$
f_{2}\left(x^{\prime}\right) / f_{1}\left(x^{\prime}\right) \leq f_{2}(x) / f_{1}(x) .
$$

With the assumption that $f_{1} / f_{2}$ is a strictly decreasing function, $x^{\prime} \leq x$.

\section{Appendix F: Differentiability of L1-SVM RM Bound: An Example}

We will demonstrate four things by the following example:

1. The original modified RM bound is not differentiable.

2. Our RM bound is differentiable.

3. Our RM bound is not twice differentiable.

4. Our RM bound is not a convex function with respect to $C$ and $\sigma^{2}$.

Given two different data points in two classes, if the RBF kernel is used, the dual problem has the following form:

$$
\min _{\alpha_{1}, \alpha_{2}} \frac{1}{2}\left[\begin{array}{ll}
\alpha_{1} & \alpha_{2}
\end{array}\right]\left[\begin{array}{cc}
1 & -a \\
-a & 1
\end{array}\right]\left[\begin{array}{l}
\alpha_{1} \\
\alpha_{2}
\end{array}\right]-e^{T} \alpha
$$

subject to $\alpha_{1}-\alpha_{2}=0$,

$$
0 \leq \alpha_{1}, \alpha_{2} \leq C
$$

where $0<a<1$.

At the optimal solution,

$$
\begin{aligned}
& \alpha_{1}=\alpha_{2}= \begin{cases}\frac{1}{1-a} & \text { if } C \geq \frac{1}{1-a}, \\
C & \text { if } 0 \leq C \leq \frac{1}{1-a},\end{cases} \\
& \xi_{1}=\xi_{2}= \begin{cases}0 & \text { if } C \geq \frac{1}{1-a}, \\
1-(1-a) C & \text { if } 0 \leq C \leq \frac{1}{1-a} .\end{cases}
\end{aligned}
$$

We have

$$
e^{T} \alpha= \begin{cases}\frac{2}{1-a} & \text { if } C \geq \frac{1}{1-a}, \\ 2 C & \text { if } 0 \leq C \leq \frac{1}{1-a},\end{cases}
$$




$$
e^{T} \xi= \begin{cases}0, & \text { if } C \geq \frac{1}{1-a}, \\ 2-2(1-a) C, & \text { if } 0 \leq C \leq \frac{1}{1-a} .\end{cases}
$$

Therefore, $e^{T} \alpha$ is not differentiable at $C=1 /(1-a)$. We then claim that $\left(R^{2}+\frac{1}{C}\right) e^{T} \alpha$ is not differentiable. Otherwise, with the fact that $R^{2}+1 / C$ is differentiable, we imply that $e^{T} \alpha$ is differentiable, a contradiction.

Now the optimization problem for finding $R^{2}$ is:

$$
\begin{aligned}
\max _{\beta_{1}, \beta_{2}} & e^{T} \beta-\frac{1}{2}\left[\begin{array}{ll}
\beta_{1} & \beta_{2}
\end{array}\right]\left[\begin{array}{ll}
1 & a \\
a & 1
\end{array}\right]\left[\begin{array}{l}
\beta_{1} \\
\beta_{2}
\end{array}\right] \\
\text { subject to } & \beta_{1}+\beta_{2}=1, \\
& 0 \leq \beta_{1}, \beta_{2} .
\end{aligned}
$$

Clearly,

$$
\beta_{1}=\beta_{2}=\frac{1}{2}, R^{2}=1-\frac{1}{2}(1+a)=\frac{1-a}{2} .
$$

Hence, equation 3.1 becomes

$$
R^{2} e^{T} \alpha+e^{T} \xi= \begin{cases}1 & \text { if } C \geq \frac{1}{1-a} \\ 2-(1-a) C & \text { if } 0 \leq C \leq \frac{1}{1-a}\end{cases}
$$

Therefore,

$$
\frac{\partial\left(R^{2} e^{T} \alpha+e^{T} \xi\right)}{\partial C}= \begin{cases}0 & \text { if } C>\frac{1}{1-a}, \\ -(1-a) & \text { if } 0 \leq C<\frac{1}{1-a},\end{cases}
$$

so $R^{2} e^{T} \alpha+e^{T} \xi$ is also not differentiable. We can further show that the original modified RM bound, $D^{2} e^{T} \alpha+\sum_{i=1}^{l} \xi_{i}$, is also not differentiable:

$$
D^{2} e^{T} \alpha+e^{T} \xi= \begin{cases}4 & \text { if } C \geq \frac{1}{1-a}, \\ 2+2(1-a) C & \text { if } 0 \leq C \leq \frac{1}{1-a},\end{cases}
$$

and

$$
\frac{\partial\left(D^{2} e^{T} \alpha+e^{T} \xi\right)}{\partial C}= \begin{cases}0 & \text { if } C>\frac{1}{1-a}, \\ 2(1-a) & \text { if } 0 \leq C<\frac{1}{1-a} .\end{cases}
$$

For our RM bound, we can easily see that it is partially differentiable:

$$
e^{T} \alpha+C \sum \xi_{i}= \begin{cases}\frac{2}{1-a} & \text { if } C \geq \frac{1}{1-a}, \\ 4 C-2(1-a) C^{2} & \text { if } 0 \leq C \leq \frac{1}{1-a} .\end{cases}
$$


Then

$$
\lim _{C \rightarrow\left(\frac{1}{1-a}\right)^{-}} \frac{\partial\left(e^{T} \alpha+C \sum \xi_{i}\right)}{\partial C}=0=\lim _{C \rightarrow\left(\frac{1}{1-a}\right)^{+}} \frac{\partial\left(e^{T} \alpha+C \sum \xi_{i}\right)}{\partial C} .
$$

With the continuity, our RM bound is differentiable. However,

$$
\begin{aligned}
& \left(R^{2}+\frac{\Delta}{C}\right)\left(\|w\|^{2}+2 C \sum \xi_{i}\right) \\
& \quad= \begin{cases}1+\frac{2 \Delta}{(1-a) C} & \text { if } C \geq \frac{1}{1-a} \\
\left(\frac{1-a}{2}+\frac{\Delta}{C}\right)\left(4 C-2(1-a) C^{2}\right) & \text { if } 0<C \leq \frac{1}{1-a}\end{cases}
\end{aligned}
$$

The second derivative is

$$
\frac{\partial^{2}\left(R^{2}+\frac{\Delta}{C}\right)\left(\|w\|^{2}+2 C \sum \xi_{i}\right)}{\partial C \partial C}= \begin{cases}\frac{4 \Delta}{1-a} C^{-3} & \text { if } C>\frac{1}{1-a}, \\ -2(1-a)^{2} & \text { if } 0<C<\frac{1}{1-a} .\end{cases}
$$

That is, at $C=1 /(1-a)$, the second derivative is not available.

Finally, for convexity, when $C \leq 1 /(1-a)$, equations F.1 and F.2 imply $f\left(C, \sigma^{2}\right)=4-(1-a)^{2} C^{2}$. When $\sigma^{2}$ is fixed, $a$ is a constant and $f$ is a concave function of $C$.

\section{Appendix G: RM Bound for L2-SVM Is Not Twice Differentiable:}

\section{An Example}

Given two points in one class and one point in the other, if the RBF kernel is used, the dual problem of L2-SVM is:

$$
\begin{aligned}
& \min _{\alpha_{1}, \alpha_{2}, \alpha_{3}} \frac{1}{2}\left[\begin{array}{lll}
\alpha_{1} & \alpha_{2} & \alpha_{3}
\end{array}\right]\left[\begin{array}{ccc}
1+\frac{1}{c} & a & -b \\
a & 1+\frac{1}{c} & -c \\
-b & -c & 1+\frac{1}{c}
\end{array}\right]\left[\begin{array}{l}
\alpha_{1} \\
\alpha_{2} \\
\alpha_{3}
\end{array}\right]-e^{T} \alpha \\
& \text { subject to } \alpha_{1}+\alpha_{2}=\alpha_{3} \text {, } \\
& 0 \leq \alpha_{1}, \alpha_{2}, \alpha_{3} \text {, }
\end{aligned}
$$

and $\tilde{R}^{2}$ is the objective value of

$$
\max _{\beta_{1}, \beta_{2}, \beta_{3}} 1+\frac{1}{C}-\left[\begin{array}{lll}
\beta_{1} & \beta_{2} & \beta_{3}
\end{array}\right]\left[\begin{array}{ccc}
1+\frac{1}{C} & a & b \\
a & 1+\frac{1}{C} & c \\
b & c & 1+\frac{1}{C}
\end{array}\right]\left[\begin{array}{l}
\beta_{1} \\
\beta_{2} \\
\beta_{3}
\end{array}\right]
$$

subject to $\beta_{1}+\beta_{2}+\beta_{3}=1$,

$$
0 \leq \beta_{1}, \beta_{2}, \beta_{3} \text {, }
$$

where $0<a, b, c<1$. 
Consider a problem satisfying the following conditions:

$$
\begin{aligned}
a+c-b-1 & >0 \\
c & >b \\
a & >b .
\end{aligned}
$$

Then the optimal $\alpha$ and $\beta$ near $C=1 /(a+c-b-1)$ are

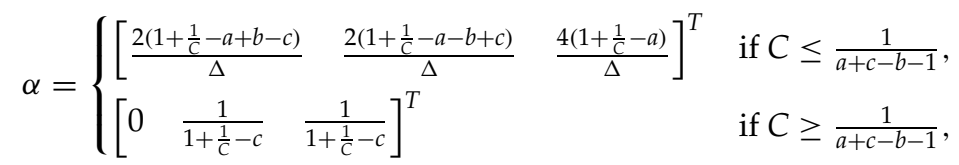

and

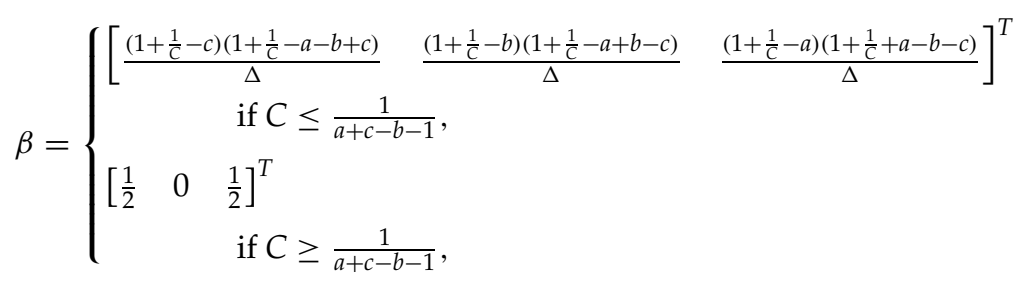

where $\Delta=3\left(1+\frac{1}{C}\right)^{2}-2(a+b+c)\left(1+\frac{1}{C}\right)-\left(a^{2}+b^{2}+c^{2}\right)+(2 a b+2 b c$ $+2 a c)$.

To have that $\tilde{R}^{2}\|\tilde{w}\|^{2}$ is not twice differentiable, it is sufficient to show that the left and right second derivatives at some $C$ are different.

Let $a=c=0.8, b=0.5$ satisfy equation G.1 and $\sigma=1 / \sqrt{2}$. This is possible because

$$
\begin{aligned}
& \left\|x_{1}-x_{2}\right\|=\sqrt{-\log a} \approx 0.4724, \\
& \left\|x_{1}-x_{3}\right\|=\sqrt{-\log b} \approx 0.8326, \\
& \left\|x_{2}-x_{3}\right\|=\sqrt{-\log c} \approx 0.4724,
\end{aligned}
$$

and $\left(x_{1}, x_{2}, x_{3}\right)$ form an isosceles triangle. Using MATLAB symbolic toolbox to calculate the left and right second derivatives at $1 / C=a+c-b-1$, we get

$$
\begin{aligned}
\lim _{C \rightarrow\left(\frac{1}{a+c-b-1}\right)^{+}} \frac{\partial^{2} \tilde{R}^{2}\|\tilde{w}\|^{2}}{\partial C^{2}} & =-\frac{1}{225}, \\
\lim _{C \rightarrow\left(\frac{1}{a+c-b-1}\right)^{-}} \frac{\partial^{2} \tilde{R}^{2}\|\tilde{w}\|^{2}}{\partial C^{2}} & =-\frac{1}{450} .
\end{aligned}
$$




\section{Acknowledgments}

This work was supported in part by the National Science Council of Taiwan via the grant NSC 90-2213-E-002-111. We thank S. Sathiya Keerthi, Jorge Moré, and Olivier Chapelle for very useful discussion. In particular, Olivier Chapelle helped to remove an assumption of theorem 1.

\section{References}

Bailey, R. R., Pettit, E. J., Borochoff, R. T., Manry, M. T., \& Jiang, X. (1993). Automatic recognition of USGS land use/cover categories using statistical and neural networks classifiers. In SPIE OE/Aerospace and Remote Sensing. Bellingham, WA: SPIE.

Bonnans, J. F., \& Shapiro, A. (1998). Optimization problems with perturbations: A guided tour. SIAM Review, 40(2), 228-264.

Chang, C.-C., \& Lin, C.-J. (2001a). IJCNN 2001 challenge: Generalization ability and text decoding. In Proceedings of IJCNN. Piscataway, NJ: IEEE.

Chang, C.-C., \& Lin, C.-J. (2001b). LIBSVM: A library for support vector machines. Available on-line: http://www.csie.ntu.edu.tw/ cjlin/libsvm.

Chang, M.-W., \& Lin, C.-J. (2003). Properties of the dual SVM solution as a function of parameters (Tech. Report). Taipei: Department of Computer Science and Information Engineering, National Taiwan University.

Chapelle, O., Vapnik, V., Bousquet, O., \& Mukherjee, S. (2002). Choosing multiple parameters for support vector machines. Machine Learning, 46, 131-159.

Cristianini, N., Campbell, C., \& Shawe-Taylor, J. (1999). Dynamically adapting kernels in support vector machines. In M. S. Kearns, S. A. Solla, \& and D. A. Cohn (Eds.), Advances in neural information processing systems, 11 (pp. 204-210). Cambridge, MA. MIT Press.

Duan, K., Keerthi, S. S., \& Poo, A. N. (2003). Evaluation of simple performance measures for tuning SVM hyperparameters. Neurocomputing, 51, 41-59.

Elisseeff, A., \& Pontil, M. (2002). Leave-one-out error and stability of learning algorithms with applications. In J. Suykens, G. Horvath, S. Basu, C. Micchelli, \& J. Vandewalle (Eds.), Learning Theory and Practice. Washington, DC: IOS Press.

Hastie, T., Tibshirani, R., \& Friedman, J. (2002). The elements of statistical learning: Data mining, inference, and prediction. Berlin: Springer-Verlag.

Jaakkola, T. S., \& Haussler, D. (1999). Probabilistic kernel regression models. In Proceedings of the 1999 Conference on AI and Statistics. Society for Artificial Intelligence in Statistics.

Joachims, T. (1998). Making large-scale SVM learning practical. In B. Schölkopf, C. J. C. Burges, \& A. J. Smola (Eds.), Advances in kernel methods-Support vector learning, Cambridge, MA: MIT Press.

Joachims, T. (2000). Estimating the generalization performance of a SVM efficiently. In Proceedings of the International Conference on Machine Learning, San Mateo: Morgan Kaufmann. 
Keerthi, S. S. (2002). Efficient tuning of SVM hyperparameters using radius/margin bound and iterative algorithms. IEEE Transactions on Neural Networks, 13, 1225-1229.

Keerthi, S. S., \& Lin, C.-J. (forthcoming). Asymptotic behaviors of support vector machines with gaussian kernel.

Li, D.-H., \& Fukushima, M. (2001). On the global convergence of the BFGS method for nonconvex unconstrained optimization problems. SIAM Journal on Optimization, 11(4), 1054-1064.

Lin, C.-J., \& Moré, J. J. (1999). Newton's method for large-scale bound constrained problems. SIAM Journal on Optimization, 9, 1100-1127.

Micchelli, C. A. (1986). Interpolation of scattered data: Distance matrices and conditionally positive definite functions. Constructive Approximation, 2, 1122.

Nocedal, J., \& Wright, S. J. (1999). Numerical optimization. New York: SpringerVerlag.

Prokhorov, D. (2001). IJCNN 2001 neural network competition. Slide presentation in IJCNN'01, Ford Research Laboratory. Available on-line: http://www.geocities.com/ijcnn/nnc ijcnn01.pdf.

Rätsch, G. (1999). Benchmark data sets. Available on-line: http://ida.first.gmd. de/ raetsch/data/benchmarks.htm.

Schölkopf, B., Burges, C., \& Vapnik, V. (1995). Extracting support data for a given task. In U. M. Fayyad \& R. Uthurusamy (Eds.), Proceedings of the First International Conference on Knowledge Discovery and Data Mining. Menlo Park, CA: AAAI Press.

Vapnik, V. (1998). Statistical learning theory. New York: Wiley.

Vapnik, V., \& Chapelle, O. (2000). Bounds on error expectation for support vector machines. Neural Computation, 12(9), 2013-2036.

Wahba, G. (1998). Support vector machines, reproducing kernel Hilbert spaces, and randomized GACV. In B. Schölkopf, C. J. C. Burges, \& A. J. Smola (Eds.), Advances in kernel methods: Support vector learning (pp. 69-88). Cambridge, MA: MIT Press.

Wahba, G., Lin, Y., \& Zhang, H. (2000). Generalized approximate cross validation for support vector machines. In A. Smola, P. Bartlett, B. Schölkopf, \& D. Schuurmans (Eds.), Advances in large margin classifiers (pp. 297-309). Cambridge, MA: MIT Press.

Zhang, T. (2001). A leave-one-out cross validation bound for kernel methods with applications in learning. In Proceedings of the 14th Annual Conference on Computational Learning Theory (pp. 427-443). Berlin: Springer-Verlag.

Received August 2, 2002; accepted April 4, 2003. 